Pacific

Journal of

Mathematics

ON THE EQUIVALENCE PROBLEM FOR TORIC CONTACT STRUCTURES ON $\mathbf{S}^{3}$-BUNDLES OVER $\mathbf{S}^{2}$

Charles P. Boyer AND Justin Pati 


\title{
ON THE EQUIVALENCE PROBLEM FOR TORIC CONTACT STRUCTURES ON $\mathbf{S}^{3}$-BUNDLES OVER $\mathbf{S}^{2}$
}

\author{
Charles P. Boyer AND Justin PATi
}

\begin{abstract}
We study the contact equivalence problem for toric contact structures on $S^{3}$ bundles over $S^{2}$. That is, given two toric contact structures, one can ask the question: when are they equivalent as contact structures while inequivalent as toric contact structures? In general this appears to be a difficult problem. To show that two toric contact structures with the same first Chern class are contact inequivalent, we use Morse-Bott contact homology. To find inequivalent toric contact structures that are contact equivalent, we show that the corresponding 3-tori belong to distinct conjugacy classes in the contactomorphism group. We treat a subclass of contact structures which includes the Sasaki-Einstein contact structures $Y^{p, q}$ studied by physicists with the anti-de Sitter/conformal field theory conjecture. In this case we give a complete solution to the contact equivalence problem by showing that $Y^{p, q}$ and $Y^{p^{\prime}, q^{\prime}}$ are inequivalent as contact structures if and only if $p \neq p^{\prime}$.
\end{abstract}

Introduction

1. Contact structures and cones

2. Toric contact structures of Reeb type

3. Contact homology

4. The contact equivalence problem

5. Applications to Sasakian geometry

Appendix: Orbifold Gromov-Witten invariants

Acknowledgements

References
277

280

285

289

304

314

318

320

320

\section{Introduction}

It is well known that contact structures have only discrete invariants; that is, Gray's theorem says that the deformation theory is trivial. Apparently, the crudest such

Boyer was partially supported by grant \#245002 from the Simons Foundation. Pati was partially supported by the Göran Gustafsson Foundation for Research in Natural Sciences and Medicine.

MSC2010: 53D10, 53D20, 53D42.

Keywords: toric contact geometry, equivalent contact structures, orbifold Hirzebruch surface, contact homology, extremal Sasakian structures. 
invariant is the first Chern class of the contact bundle $\mathcal{D}$. Indeed, the mod 2 reduction of $\mathcal{D}$ is a topological invariant, namely the second Stiefel-Whitney class. A much more subtle and powerful invariant is contact homology, a small part of the more general symplectic field theory (SFT) of Eliashberg, Givental, and Hofer [Eliashberg et al. 2000] — which can be used to distinguish contact structures belonging to the same isomorphism class of oriented $2 n$-plane bundle.

On the other hand, given two contact structures with the same invariants, when can one show that they are equivalent? In full generality this appears to be a very difficult problem. However, if we restrict ourselves to toric contact structures in dimension five, we can begin to get a handle on things. The problem is of particular interest when applied to toric contact manifolds since they have been classified [Lerman 2003a]. Thus, one is interested in when two inequivalent toric contact structures are equivalent as contact structures. Specializing further we consider all toric contact structures on $S^{3}$-bundles over $S^{2}$. It is well known that such manifolds are classified by $\pi_{1}(\mathrm{SO}(4))=\mathbb{Z}_{2}$, so there are exactly two such bundles, the trivial bundle $S^{2} \times S^{3}$ and one nontrivial bundle $X_{\infty}$ (in the notation of [Barden 1965]). They are distinguished by their second Stiefel-Whitney class $w_{2} \in H^{2}\left(M, \mathbb{Z}_{2}\right)$. The problem of determining when two such toric contact structures belong to equivalent contact structures is now somewhat tractable owing to the work of Karshon [2003] and Lerman [2003b].

The general toric contact structures on $S^{2} \times S^{3}$ or $X_{\infty}$ depend on four integers $\left(p_{1}, p_{2}, p_{3}, p_{4}\right)$ that satisfy $\operatorname{gcd}\left(p_{i}, p_{j}\right)=1$ for $i=1,2$ and $j=3,4$. We write the contact structures as $\mathcal{D}_{\boldsymbol{p}}$, using vector notation $\boldsymbol{p}$ for the quadruple. However, this general situation appears somewhat intractable, so we consider the special case when $p_{1}=p_{2}$ or $p_{3}=p_{4}$, which is more tractable because then a certain quotient is a Hirzebruch surface with branch divisors. We may as well assume that $p_{3}=p_{4}$. It is often convenient to further divide this case into two subcases, as follows. We set $\boldsymbol{p}=(j, 2 k-j, l, l)$ for $S^{2} \times S^{3}$ and $\boldsymbol{p}=(j, 2 k-j+1, l, l)$ for $X_{\infty}$ with $1 \leq j \leq k$. We denote either one of these contact structures by $\mathcal{D}_{p_{1}, p_{2}, l, l}, \mathcal{D}_{j, 2 k-j, l, l}, \mathcal{D}_{j, 2 k-j+1, l, l}$ or simply as $\mathcal{D}_{j, k, l}$, depending on which notation is more convenient. The first Chern class $c_{1}(\mathcal{D})$ of the contact bundle is a classical algebraic invariant of the contact structure. For the contact structure $\mathcal{D}_{\boldsymbol{p}}$ we will give an explicit formula for $c_{1}\left(\mathcal{D}_{p}\right)$ which for $\mathcal{D}_{j, 2 k-j, l, l}$ and $\mathcal{D}_{j, 2 k-j+1, l, l}$ equals $2(k-l)$ and $2(k-l)+1$, respectively. Using contact homology we show that even if $\mathcal{D}_{j, k, l}$ and $\mathcal{D}_{j^{\prime}, k^{\prime}, l^{\prime}}$ are not distinguished by the first Chern class, they are inequivalent if $k \neq k^{\prime}$.

Our main result about inequivalence is this:

Theorem 1. The two toric contact structures $\mathcal{D}_{p_{1}, p_{2}, l, l}$ and $\mathcal{D}_{p_{1}^{\prime}, p_{2}^{\prime}, l^{\prime}, l^{\prime}}$ on $S^{2} \times S^{3}$ or $X_{\infty}$ are inequivalent contact structures if $p_{1}^{\prime}+p_{2}^{\prime} \neq\left(p_{1}+p_{2}\right)$. 
For our main result about equivalence, we need to specialize a bit further. In this case we require that $\operatorname{gcd}\left(p_{2}-p_{1}, l\right)$ be constant. We have

Theorem 2. The two contact structures $\mathcal{D}_{p_{1}, p_{2}, l, l}$ and $\mathcal{D}_{p_{1}^{\prime}, p_{2}^{\prime}, l, l}$ satisfying $p_{1}^{\prime}+p_{2}^{\prime}=$ $p_{1}+p_{2}$ are equivalent if $\operatorname{gcd}\left(l, p_{2}-p_{1}\right)=\operatorname{gcd}\left(l, p_{2}^{\prime}-p_{1}^{\prime}\right)$.

Recently there has been a great deal of focus on certain toric contact structures $Y^{p, q}$ with vanishing first Chern class on $S^{2} \times S^{3}$ discovered by Gauntlett, Martelli, Sparks, and Waldram [2004a], and used in their study of the anti-de Sitter/conformal field theory conjecture [Gauntlett et al. 2004b; 2005] (see Chapter 11 of [Boyer and Galicki 2008] and [Sparks 2011] and references therein). In our notation the contact structures $Y^{p, q}$ correspond to $\mathcal{D}_{p-q, p+q, p, p}$. Remarkably our results give a complete answer to the contact equivalence problem for these structures.

Theorem 3. Let $\phi$ denote the Euler $\phi$-function. The toric contact structures $Y^{p, q}$ and $Y^{p^{\prime}, q^{\prime}}$ on $S^{2} \times S^{3}$ belong to equivalent contact structures if and only if $p^{\prime}=p$, and for each fixed integer $p>1$ there are exactly $\phi(p)$ toric contact structures $Y^{p, q}$ on $S^{2} \times S^{3}$ that are equivalent as contact structures, denoted by $\mathcal{D}_{p}$. Moreover, the contactomorphism group of $\mathcal{D}_{p}$ has at least $\phi(p)$ conjugacy classes of maximal tori of dimension three.

A partial result, namely that $Y^{p^{\prime}, 1}$ and $Y^{p, 1}$ are inequivalent contact structures if $p^{\prime} \neq p$, was recently given by Abreu and Macarini [2012], and an outline of the proof of Theorem 3 was recently given by one of us [Boyer 2011a].

As a bonus we also obtain the following results concerning extremal Sasakian structures:

Corollary 4. For each such contact structure $\mathcal{D}_{p}$ there are $\phi(p)$ compatible SasakiEinstein metrics that are inequivalent as Riemannian metrics.

Corollary 5. For both $S^{2} \times S^{3}$ and $X_{\infty}$ the moduli space of extremal Sasakian structures has a countably infinite number of components. Moreover, each component has extremal Sasakian metrics of positive Ricci curvature whose isometry group contains $T^{3}$.

This corollary follows already from the results of [Pati 2009; 2010; Boyer 2011b], but Theorem 1 actually gives a much larger class in the sense that there are countably many new components. As shown in [Boyer 2011b], many of these components are themselves non-Hausdorff.

Corollary 6. The moduli space of Sasaki-Einstein metrics on $S^{2} \times S^{3}$ has a countably infinite number of components. Moreover, each such component has SasakiEinstein metrics whose isometry group contains $T^{3}$.

This corollary also follows from [Abreu and Macarini 2012]. 


\section{Contact structures and cones}

It is well known that contact geometry is equivalent to the geometry of certain symplectic cones. However, for certain contact structures there are several cones that become important, and as we shall see they are all related.

A warning about notation. In contact topology the contact bundle is usually denoted by $\xi$, whereas in Sasakian geometry $\xi$ is almost always a Reeb vector field. To avoid confusion we eschew the use of $\xi$ completely, and use $\mathcal{D}$ for the contact bundle and $R$ for a Reeb vector field.

Contact structures. Recall that a contact structure ${ }^{1}$ on a connected oriented manifold $M$ is an equivalence class of 1 -forms $\eta$ satisfying $\eta \wedge(d \eta)^{n} \neq 0$ everywhere on $M$ where two 1-forms $\eta$ and $\eta^{\prime}$ are equivalent if there exists a nowhere-vanishing function $f$ such that $\eta^{\prime}=f \eta$. We shall also assume that our contact structure has an orientation, or equivalently, the function $f$ is everywhere positive. More conveniently the contact structure can be thought of as the oriented $2 n$-plane bundle defined by $\mathcal{D}=\operatorname{ker} \eta$, and we denote by $\mathfrak{C}^{+}(\mathcal{D})$ the set of all contact 1 -forms representing the oriented bundle $\mathcal{D}$.

Recall that an almost-complex structure $J$ on $\mathcal{D}$ is compatible with the contact structure if these two conditions hold for any smooth sections $X$ and $Y$ of $\mathcal{D}$ :

$$
d \eta(J X, J Y)=d \eta(X, Y), \quad d \eta(J X, Y)>0 .
$$

It is easy to see that these conditions are independent of the choice of 1 -form $\eta$ representing $\mathcal{D}$. The space of almost-complex structures that are compatible with $\mathcal{D}$ is contractible which implies that the Chern classes are invariants of the contact bundle $\mathcal{D}$. In particular, the first Chern class $c_{1}(\mathcal{D})$ will play an important role for us. Notice also that the pair $(\mathcal{D}, J)$ defines a strictly pseudoconvex almost-CR structure on $M$, and a choice of contact form $\eta$ gives a choice of Levi form - essentially $d \eta$.

Also for every choice of contact 1-form $\eta$ there exists a unique vector field $R$, called the Reeb vector field, that satisfies $\eta(R)=1$ and $R\lrcorner d \eta=0$. Such vector fields and the orbits of their flows will play a crucial role for us. We can now extend $J$ to an endomorphism $\Phi$ of $T M$ by defining $\left.\Phi\right|_{\mathcal{D}}=J$ and $\Phi R=0$. The triple $(R, \eta, \Phi)$ canonically defines a Riemannian metric on $M$ by setting $g=d \eta \circ(\Phi \otimes \mathbb{1})+\eta \otimes \eta$, and the quadruple $(R, \eta, \Phi, g)$ is known as a contact metric structure on $M$.

Notice that $R$ defines a one-dimensional foliation $\mathcal{F}_{R}$ on $M$, often called the characteristic foliation. We say that the foliation $\mathcal{F}_{R}$ is quasiregular if there is a positive integer $k$ such that each point has a foliated coordinate chart $(U, x)$ such that each leaf of $\mathcal{F}_{R}$ passes through $U$ at most $k$ times. If $k=1$ then the foliation is

${ }^{1}$ This is not the most general definition of a contact structure, but it suffices in most situations (compare [Boyer and Galicki 2008, Chapter 6]), and certainly for us here. 
called regular. We also say that the corresponding contact 1-form $\eta$ is quasiregular (regular), and more generally that a contact structure $\mathcal{D}$ is quasiregular (regular) if it has a quasiregular (regular) contact 1-form. A contact 1-form (or characteristic foliation) that is not quasiregular is called irregular. On a compact manifold any quasiregular contact form is necessarily $K$-contact, and then the foliation $\mathcal{F}_{R}$ is equivalent to a locally free circle action (compare [Boyer and Galicki 2008, §7.1]) preserving the quadruple $(R, \eta, \Phi, g)$. This is the case that we are interested in. The quotient space $\mathcal{Z}=M / \mathcal{F}_{R}$ is a compact orbifold with a naturally defined symplectic structure $\omega$ and compatible almost-complex structure $\hat{J}$ satisfying $\pi^{*} \omega=d \eta$ and $J$ is the horizontal lift of $\hat{J}$, that is, $(\omega, \hat{J})$ defines an almost-Kähler structure on the orbifold $z$. Moreover, $\eta$ can be interpreted as a connection 1-form in the principal $S^{1}$ orbibundle $\pi: M \rightarrow Z$ with curvature 2 -form $\pi^{*} \omega$.

In this paper we are interested in the case when both $J$ and $\hat{J}$ are integrable. Then the quadruple $(R, \eta, \Phi, g)$ is a Sasakian structure on $M$, and $(\omega, \hat{J})$ defines a is projective algebraic orbifold structure on $Z$ with an orbifold Kähler metric. This construction has a converse, that is, beginning with a compact almost-Kähler orbifold one can construct a $K$-contact structure on the total space of a certain $S^{1}$ orbibundle over $Z$. This is often referred to as the orbifold Boothby-Wang construction. ${ }^{2}$ It lies at the heart of the proof of Theorem 2. Indeed, we shall show the equivalence of certain contact structures by exhibiting a symplectomorphism between their corresponding quotient orbifolds.

Orbifolds. As just described, orbifolds will play an important role for us in this paper. We refer to [Boyer and Galicki 2008, Chapter 4] for the basic definitions and results. Here we want to emphasize several aspects. First, many cohomology classes that are integral classes on manifolds are only rational classes on the underlying topological space of an orbifold, in particular, the orbifold first Chern class of a complex line orbibundle or circle orbibundle is generally a rational class. However, not all rational classes occur as such. To determine which rational classes can be used to classify line orbibundles, it is convenient to pass to Haefliger's classifying space $B X$ (see [Haefliger 1984] and/or [Boyer and Galicki 2008, Chapter 4]) of an orbifold $X$ where, as with smooth manifolds, all complex line orbibundles correspond to integral cohomology classes. Let $\mathcal{X}$ be a complex orbifold with underlying topological space $X$. Then Haefliger's orbifold cohomology $H_{\text {orb }}^{*}(X, \mathbb{Z})$ equals $H^{*}(B X, \mathbb{Z})$, which is generally different than $H^{*}(X, \mathbb{Z})$, but satisfies $H_{\text {orb }}^{*}(X, \mathbb{Z}) \otimes \mathbb{Q}=H^{*}(X, \mathbb{Z}) \otimes \mathbb{Q}$. So, for example, we obtain an integral cohomology class $p^{*} c_{1}^{\text {orb }}(X) \in H_{\text {orb }}^{2}(X, \mathbb{Z})$ for complex line orbibundles from the rational class $c_{1}^{\text {orb }}(X) \in H^{2}(X, \mathbb{Q})$. This amounts to clearing the order of the orbifold in the denominator. Here $p: B X \rightarrow X$ is the

${ }^{2}$ In [Eliashberg et al. 2000] a contact manifold constructed in this way is called a prequantization space. 
natural projection. We warn the reader that the orbifold cohomology $H_{\mathrm{orb}}^{*}(X, \mathbb{Z})$ is not the Chen-Ruan cohomology.

The orbifolds that occur in this paper are of a special type. They are all complex orbifolds whose underlying space is a smooth projective algebraic variety with an added orbifold structure. In such cases it is convenient to view an orbifold $X$ as a pair $(X, \Delta)$ where $X$ is a smooth algebraic variety and $\Delta$ is a certain $\mathbb{Q}$-divisor, called a branch divisor [Boyer et al. 2005; Ghigi and Kollár 2007; Boyer and Galicki 2008, Chapter 4]. We write $(X, \varnothing)$ to denote the algebraic variety $X$ with the trivial orbifold structure, that is, the charts are just the standard manifold charts. In this situation, as emphasized in [Ghigi and Kollár 2007], we consider the map $\mathbb{1}_{X}:(X, \Delta) \rightarrow(X, \varnothing)$, which is the identity as a set map, and a Galois cover with trivial Galois group.

Symplectic cones. Given a contact structure $\mathcal{D}$ on $M$ we recall the symplectic cone $C(M)=M \times \mathbb{R}^{+}$with its natural symplectic structure (the symplectization of $(M, \mathcal{D})) \Omega=d\left(r^{2} \eta\right)$, where $r$ is a coordinate on $\mathbb{R}^{+}$. Recall the Liouville vector field $\Psi=r \partial / \partial r$ on the cone $C(M)$.

Now for each choice of contact form $\eta \in \mathfrak{C}^{+}(\mathcal{D})$ there is a natural extension of the almost-complex structure $J$ on $\mathcal{D}$ to an almost-complex structure $I$ on the cone $C(M)$ defined uniquely by

$$
I=\Phi+\Psi \otimes \eta, \quad I \Psi=-R,
$$

where $\Phi$ is the extension of $J$ to $T M$ defined by $\Phi R=0$. We can also check that there is a one-to-one correspondence between the compatible almost-complex structures $I$ on $C(M)$ and elements of $\mathfrak{C}^{+}(\mathcal{D})$, and that (1) hold, so we recover the full-contact metric structure for each $\eta \in \mathfrak{C}^{+}(\mathcal{D})$. Given an almost-complex manifold, $W$, with complex structure $j$, a $C^{\infty}$ map, $u$, from $W$ into the almostcomplex manifold $(N, J)$ is called $J$-holomorphic if $d u+J(u) d(u \circ j)=0$. We are specifically interested in pseudoholomorphic maps into the cone, that is, maps which are pseudoholomorphic with respect to the almost-complex structure given by (1). Such maps from a Riemann surface into $C(M)$ are of particular interest and are known as pseudoholomorphic curves.

Summarizing, we have these correspondences:

(1) symplectic cone $(C(M), \Omega) \leftrightarrow$ contact structure $(M, \mathcal{D})$,

(2) almost-Kähler cone $(C(M), \Omega, I) \leftrightarrow$ contact metric structure $(M, R, \eta, \Phi, g)$,

(3) almost-Kähler cone $(C(M), \Omega, I)$ with $\Psi-i R$ pseudoholomorphic $\leftrightarrow K$-contact structure $(M, R, \eta, \Phi, g)$, and

(4) Kähler cone $(C(M), \Omega, I)$ with $\Psi-i R$ holomorphic $\leftrightarrow$ Sasakian structure $(M, R, \eta, \Phi, g)$. 
Remark 1.1. In the sequel when we study pseudoholomorphic curves, it is customary to parametrize the cone by $\mathbb{R}$ so that the singularity, which appears at 0 in the parametrization above, appears at $-\infty$ instead. This amounts to choosing $\Omega=d\left(e^{r} \eta\right)$ for the symplectic form on $C(M)$.

Sasakian structures. The contact structures considered in this paper are all of Sasaki type, that is, there is a contact form $\eta$ and compatible metric $g$ such that $\mathcal{S}=(R, \eta, \Phi, g)$ is a Sasakian structure on $M$. In this case not only is the cone $C(M)$ discussed above Kähler, but the geometry transverse to the characteristic foliation $\mathcal{F}_{R}$ is also Kähler. This gives rise to a basic cohomology $\operatorname{ring} H_{B}^{*}\left(\mathcal{F}_{R}\right)$ (see [Boyer and Galicki 2008, §7.2]), and a transverse Hodge theory. This gives basic Chern classes $c_{k}\left(\mathcal{F}_{R}\right)$ which, if $(R, \eta, \Phi, g)$ is quasiregular, are the pullbacks of the orbifold Chern classes $c_{k}^{\text {orb }}(Z)$ on the base orbifold $Z$. In particular we are interested in the basic first Chern class $c_{1}\left(\mathcal{F}_{R}\right) \in H_{B}^{1,1}\left(\mathcal{F}_{R}\right)$. A Sasakian structure $\mathcal{S}=(R, \eta, \Phi, g)$ is said to be positive (negative) if its basic first Chern class $c_{1}\left(\mathcal{F}_{R}\right)$ can be represented by a positive (negative)-definite $(1,1)$-form. It is null if $c_{1}\left(\mathcal{F}_{R}\right)=0$, and indefinite otherwise. It follows from [Boyer 2011b, Lemma 5.1] that all Sasakian structures occurring in toric contact structures of Reeb type are either positive or indefinite. We mention that these types occur in rays, that is, performing a transverse homothety (see [Boyer and Galicki 2008, p. 228]), preserves the type.

The Sasaki cone. Let $\mathfrak{C} R(\mathcal{D}, J)$ denote the group of almost-CR transformations of $(\mathcal{D}, J)$ on $M$. If $M$ is compact, it is a Lie group which is compact except when $(\mathcal{D}, J)$ is the standard CR structure on the sphere $S^{2 n+1}$ by, in various stages, a theorem of Frances, Lee, and Schoen (see [Boyer 2013]). We let $\mathfrak{c r}(\mathcal{D}, J)$ denote the Lie algebra of $\mathfrak{C} \mathfrak{R}(\mathcal{D}, J)$. Recall [Boyer et al. 2008] that the subset

$$
\mathfrak{c r}^{+}(\mathcal{D}, J)=\{X \in \mathfrak{c r}(\mathcal{D}, J) \mid \eta(X)>0\}
$$

is independent of the choice of $\eta \in \mathfrak{C}^{+}(\mathcal{D})$ and is an open convex cone (without the cone point) in $\operatorname{cr}(\mathcal{D}, J)$. Now the adjoint action of the group $\mathfrak{C} \mathfrak{R}(\mathcal{D}, J)$ on its Lie algebra leaves $\mathfrak{c r}^{+}(\mathcal{D}, J)$ invariant, and the quotient space

$$
\kappa(\mathcal{D}, J)=\mathfrak{c r}^{+}(\mathcal{D}, J) / \mathfrak{C} \mathfrak{R}(\mathcal{D}, J)
$$

is known as the (reduced) Sasaki cone of $(\mathcal{D}, J)$. One should think of $\kappa(\mathcal{D}, J)$ as the moduli space of $K$-contact structures associated to the strictly pseudoconvex almost-CR structure $(\mathcal{D}, J)$. In the case that the almost-CR structure is integrable, $\kappa(\mathcal{D}, J)$ is the moduli space of Sasakian structures associated to $(\mathcal{D}, J)$. It is often convenient to work with the unreduced Sasaki cone given by choosing a maximal torus $T$ of $\mathfrak{C} \mathfrak{R}(\mathcal{D}, J)$. Then the unreduced Sasaki cone is $\mathfrak{t}^{+}(\mathcal{D}, J)=\mathfrak{t} \cap \mathfrak{c r} \mathfrak{r}^{+}(\mathcal{D}, J)$ where $t$ is the Lie algebra of $T$. 
Of course, many contact structures do not have a Sasaki cone. In fact, a contact structure has a nonempty Sasaki cone if and only if it is of $K$-contact type. It is important to realize that the Sasaki cone depends on the choice of transverse almost-complex structure $J$. Indeed by changing $J$ in a given $K$-contact structure, we can have more than one Sasaki cone. These occur in bouquets related to the conjugacy classes of maximal tori in the contactomorphism $\operatorname{Con}(M, \mathcal{D})$ of $(M, \mathcal{D})$ [Boyer 2011b; 2013].

The moment cone. Now let $T$ be a torus subgroup of $\mathfrak{C o n}(M, \mathcal{D})$, and let $\mathfrak{t}$ be its Lie algebra. Consider the annihilator $\mathcal{D}^{o}$ of $\mathcal{D}$ which is a trivial real line bundle over $M$. The orientation on $\mathcal{D}$ allows us to write $\mathcal{D}^{o} \backslash\{0\}=\mathcal{D}_{+}^{o} \cup \mathcal{D}_{-}^{o}$, and we can identify $\mathcal{D}_{+}^{o} \approx M \times \mathbb{R}^{+}=C(M)$. Then the contact moment map $\Upsilon: \mathcal{D}_{+}^{o} \rightarrow \mathrm{t}^{*}$ is defined by

$$
\langle\Upsilon(x, p), \tau\rangle=\left\langle p, \tau_{x}\right\rangle,
$$

where $\tau \in \mathfrak{t}$ and $\tau_{x}$ denotes the fundamental vector field associated to $\tau$ at the point $x$. The moment cone $C(\Upsilon)$ is defined [Lerman 2003a] as the union of the image set with the cone point, that is,

$$
C(\Upsilon)=\Upsilon\left(\mathcal{D}_{+}^{o}\right) \cup\{0\}
$$

By averaging over $T$ we can choose a $T$-invariant contact form $\eta$ which gives an equivariant moment map $\mu_{\eta}: M \rightarrow \mathfrak{t}^{*}$ satisfying

$$
\mu_{\eta}=\Upsilon \circ \eta
$$

Again by averaging we can choose an almost-complex structure $J$ that is $T$-invariant, so $\mathfrak{t}$ is an Abelian subalgebra of $\mathfrak{c r}(\mathcal{D}, J)$. Furthermore, the contact form $\eta$ is $K$-contact (with respect to $J$ ) if and only if its Reeb vector field $R_{\eta}$ lies in the Lie algebra $t$. In this case we also say that the torus action is of Reeb type [Boyer and Galicki 2000a]. It is easy to see that this is equivalent to the existence of an element $\tau \in \mathfrak{t}$ such that $\eta(\tau)$ is strictly positive on $M$. When the contact structure $\mathcal{D}$ is of Reeb type $C(\Upsilon)$ is a convex rational polyhedral cone, and we have the following result of Lerman [2003a].

Lemma 1.2. A T-invariant contact form $\eta$ is $K$-contact if and only if the image $\mu_{\eta}(M)$ lies in the intersection of a hyperplane $H_{\eta}$ with the moment cone $C(\Upsilon)$. Moreover, in the K-contact case the intersection $P_{\eta}=H_{\eta} \cap C(\Upsilon)$ is a simple convex polytope which is rational if and only if $\eta$ is quasiregular.

The hyperplane $H_{\eta}$ is called the characteristic hyperplane. 


\section{Toric contact structures of Reeb type}

Toric contact structures on manifolds of dimension greater than three come in two types, those where the action of the torus is free, and those where it is not [Banyaga and Molino 1993; Lerman 2003a]. The latter contain an important special subclass known as toric contact structures of Reeb type [Boyer and Galicki 2000a]. These are precisely the toric contact where the torus action is not free and the moment cone contains no nonzero linear subspace. When the moment cone contains a nontrivial linear subspace, the toric contact manifold will have infinite fundamental group. Thus, any toric contact structure on an $S^{3}$-bundle over $S^{2}$ must be of Reeb type, and these correspond precisely to convex polyhedral cones in the dual of the Lie algebra of the torus that are cones over a polytope [Boyer and Galicki 2000a; Lerman 2003a].

Definition 2.1. A toric contact manifold $(M, \mathcal{D}, \mathcal{A})$ is a contact manifold of dimension $2 n+1$ together with an effective action of a torus $T$ of dimension $n+1$ that leaves the contact structure invariant, that is, if $\mathcal{A}: T \times M \rightarrow M$ denotes the action map then $\mathcal{A}_{*} \mathcal{D}=\mathcal{D}$.

By averaging over $T$ we can always find a contact 1 -form $\eta$ representing $\mathcal{D}$ such that $\mathcal{A}^{*} \eta=\eta$. In this case we also have $\mathcal{A}_{*} R=R$ for the Reeb vector field. A toric contact manifold is said to be of Reeb type if there is a contact form $\eta \in \mathfrak{C}^{+}(\mathcal{D})$ whose Reeb vector field lies in the Lie algebra $\mathfrak{t}$ of $T$.

Two toric contact manifolds $(M, \mathcal{D}, \mathcal{A})$ and $\left(M^{\prime}, \mathcal{D}^{\prime}, \mathcal{A}^{\prime}\right)$ are said to be equivariantly equivalent (or equivalent toric contact manifolds) if there exists a contactomorphism between them that conjugates the torus actions $\mathcal{A}$ and $\mathcal{A}^{\prime}$. Toric contact manifolds were classified in [Lerman 2003a]. In this paper we are interested in inequivalent toric contact manifolds that are equivalent as contact manifolds. In this case the tori generated by the actions $\mathcal{A}$ and $\mathcal{A}^{\prime}$ belong to distinct conjugacy classes in the contactomorphism group $\mathfrak{C o n}(M, \mathcal{D})$. Furthermore, to each such conjugacy class there is an associated toric CR structure $(\mathcal{D}, J)$ which by [Boyer 2013, Theorem 7.6] is unique up to biholomorphism.

Contact reduction. It is well known (see [Boyer and Galicki 2000a; Lerman 2003a]) that every contact toric structure of Reeb type can be obtained by symmetry reduction of the standard sphere by a compact Abelian group $A$, and that this is equivalent to the symplectic reduction of the standard symplectic structure on $\mathbb{C}^{N} \backslash\{0\}$ by a compact Abelian group which commutes with the action of dilations of the cone. For this one must choose the zero level set of the toral moment map. This equivalence can be described by the commutative diagram

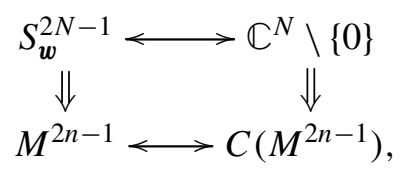


with $\operatorname{dim} A=N-n$. See [Boyer and Galicki 2008, p. 293].

Lemma 2.2. Let $M$ be an $S^{3}$-bundle over $S^{2}$. Every toric contact structure on $M$ can be obtained by contact circle reduction of the standard contact structure on $S^{7}$.

Proof. As stated above, every compact toric contact manifold of Reeb type can be obtained by symmetry reduction of the standard sphere $S^{2 N-1}$ by a compact Abelian group $A$ [Boyer and Galicki 2000a; Lerman 2003a]. Now by the homotopy exact sequence $M$ is simply connected and $\pi_{2}(M)=\mathbb{Z}$. By a result of Lerman [2004] $\pi_{1}(M)=\pi_{0}(A)$ and $\pi_{2}(M)=\pi_{1}(A)=\mathbb{Z}^{\operatorname{dim} T}$. Thus, $A$ is a torus of dimension one, that is, a circle. Since $n=3, N=n+\operatorname{dim} A=4$, so $M$ is obtained by contact reduction from $S^{7}$.

Remark 2.3. It is well known that there are exactly two $S^{3}$-bundles over $S^{2}$, distinguished by their second Stiefel-Whitney class, the trivial bundle $S^{3} \times S^{2}$ and the nontrivial bundle denoted by $X_{\infty}$ in the Barden-Smale classification [Barden 1965] of simply connected 5-manifolds. We will show their relation with the reduction parameters in Theorem 2.6.

We now describe this reduction. First, the standard $T^{4}$ action on $\mathbb{C}^{4}$ is $z_{j} \mapsto e^{i \theta_{j}} z_{j}$, and its moment map $\Upsilon_{4}: \mathbb{C}^{4} \backslash\{0\} \rightarrow \mathfrak{t}_{4}^{*}=\mathbb{R}^{4}$ is given by

$$
\Upsilon_{4}(z)=\left(\left|z_{1}\right|^{2},\left|z_{2}\right|^{2},\left|z_{3}\right|^{2},\left|z_{4}\right|^{2}\right) .
$$

Now we consider the circle group $T(\boldsymbol{p})$ acting on $\mathbb{C}^{4} \backslash\{0\}$ by

$$
\left(z_{1}, z_{2}, z_{3}, z_{4}\right) \mapsto\left(e^{i p_{1} \theta} z_{1}, e^{i p_{2} \theta} z_{2}, e^{-i p_{3} \theta} z_{3}, e^{-i p_{4} \theta} z_{4}\right),
$$

where $\boldsymbol{p}$ denotes the quadruple $\left(p_{1}, p_{2}, p_{3}, p_{4}\right)$ with $p_{i} \in \mathbb{Z}^{+}$and we assume $\operatorname{gcd}\left(p_{1}, p_{2}, p_{3}, p_{4}\right)=1$. We have an exact sequence of commutative Lie algebras

$$
0 \rightarrow \mathfrak{t}_{1}(\boldsymbol{p}) \rightarrow \mathbb{R}^{4} \stackrel{\tilde{\tilde{\omega}}}{\rightarrow} \mathfrak{t}_{3}(\boldsymbol{p}) \rightarrow 0
$$

where $\mathfrak{t}_{1}(\boldsymbol{p})$ is the Lie algebra of $T(\boldsymbol{p})$ generated by the vector field $L_{\boldsymbol{p}}=p_{1} H_{1}+$ $p_{2} \mathrm{H}_{2}-p_{3} \mathrm{H}_{3}-\mathrm{p}_{4} \mathrm{H}_{4}$.

Dualizing (8) gives

$$
0 \rightarrow \mathfrak{t}_{3}^{*}(\boldsymbol{p}) \stackrel{\tilde{\omega}^{*}}{\rightarrow}\left(\mathbb{R}^{4}\right)^{*} \rightarrow \mathfrak{t}_{1}^{*}(\boldsymbol{p}) \rightarrow 0 .
$$

The moment map $\Upsilon_{1}: \mathbb{C}^{4} \backslash\{0\} \rightarrow \mathrm{t}_{1}^{*}=\mathbb{R}$ for this action is given by

$$
\Upsilon_{1}(z)=p_{1}\left|z_{1}\right|^{2}+p_{2}\left|z_{2}\right|^{2}-p_{3}\left|z_{3}\right|^{2}-p_{4}\left|z_{4}\right|^{2} .
$$

Now consider the 1-form

$$
\eta_{0}=-\frac{i}{2} \sum_{j=0}^{n}\left(z_{j} d \bar{z}_{j}-\bar{z}_{j} d z_{j}\right)
$$


on $\mathbb{C}^{4} \backslash\{0\}$ together with the vector field

$$
R_{p}=\sum_{j} p_{j} H_{j}
$$

where $H_{j}=-i\left(z_{j} \partial / \partial z_{j}-\bar{z}_{j} \partial / \partial \bar{z}_{j}\right)$. Imposing the constraint $\eta_{0}\left(R_{\boldsymbol{p}}\right)=1$ gives $S^{7}$ represented as $\sum_{j} p_{j}\left|z_{j}\right|^{2}=1$. Then $\eta_{0}$ pulls back to a contact form on $S^{7}$, also denoted by $\eta_{0}$, with Reeb vector field $R_{\boldsymbol{p}}=p_{1} H_{1}+p_{2} H_{2}+p_{3} H_{3}+p_{4} H_{4}$. By a change of coordinates one easily sees that this represents the standard contact structure on $S^{7}$.

So the zero level set $\Upsilon_{1}^{-1}(0)$ is diffeomorphic to a cone over $S^{3} \times S^{3}$, or equivalently restricting to $S^{7}$, the zero level set of $\mu_{\eta_{0}}$ is $S^{3} \times S^{3}$, represented by

$$
p_{1}\left|z_{1}\right|^{2}+p_{2}\left|z_{2}\right|^{2}=\frac{1}{2}, \quad p_{3}\left|z_{3}\right|^{2}+p_{4}\left|z_{4}\right|^{2}=\frac{1}{2} .
$$

The action of $T(\boldsymbol{p})$ is free on this zero set if and only if $\operatorname{gcd}\left(p_{i}, p_{j}\right)=1$ for $i=1,2$ and $j=3,4$. So assuming these gcd conditions our reduced contact manifold is the $M_{\boldsymbol{p}}=\left(S^{3} \times S^{3}\right) / T(\boldsymbol{p})$ whose contact form is the unique 1-form $\eta_{\boldsymbol{p}}$ satisfying $\iota^{*} \eta_{0}=\rho^{*} \eta_{\boldsymbol{p}}$, where $\iota: \mu_{\eta_{0}}^{-1}(0) \rightarrow S^{7}$ and $\rho: \mu_{\eta_{0}}^{-1}(0) \rightarrow M_{\boldsymbol{p}}$ are the natural inclusion and projection, respectively. In order to identify $M_{p}$ we consider the $T^{2}(\boldsymbol{p})$ action on $\mu_{\eta_{0}}^{-1}(0) \approx S^{3} \times S^{3}$ generated by the $S^{1}$ action (7) together with the $S^{1}$ action generated by the Reeb vector field $R_{p}$. To guarantee a smooth quotient we have:

Definition 2.4. We say that the quadruple $\boldsymbol{p}=\left(p_{1}, p_{2}, p_{3}, p_{4}\right)$ of positive integers is admissible if $\operatorname{gcd}\left(p_{i}, p_{j}\right)=1$ for $i=1,2$ and $j=3,4$. We denote the set of admissible quadruples by $\mathcal{A}$.

Let us describe some obvious equivalences. We can interchange the coordinates $z_{1} \leftrightarrow z_{2}$, likewise $z_{3} \leftrightarrow z_{4}$. Thus, without loss of generality we can assume that $p_{1} \leq p_{2}$ and $p_{3} \leq p_{4}$. We can also interchange the pairs $\left(z_{1}, z_{2}\right)$ and $\left(z_{3}, z_{4}\right)$. It is also convenient to set $k=\operatorname{gcd}\left(p_{1}, p_{2}\right)$ and $l=\operatorname{gcd}\left(p_{3}, p_{4}\right)$ and define $\left(p_{1}, p_{2}\right)=$ $k\left(\bar{p}_{1}, \bar{p}_{2}\right)$ and $\left(p_{3}, p_{4}\right)=l\left(\bar{p}_{3}, \bar{p}_{4}\right)$ with $\operatorname{gcd}\left(\bar{p}_{1}, \bar{p}_{2}\right)=\operatorname{gcd}\left(\bar{p}_{3}, \bar{p}_{4}\right)=1$. Note that $\boldsymbol{p} \in \mathcal{A}$ implies $\operatorname{gcd}(k, l)=1$. We will need the "standard" Kähler forms on the weighted projective spaces for which we take the Bochner-flat Kähler forms of area 2 described in [Bryant 2001; David and Gauduchon 2006; Gauduchon 2009]. We denote the corresponding Kähler forms by $\omega_{\bar{p}_{1}, \bar{p}_{2}}$ and $\omega_{\bar{p}_{3}, \bar{p}_{4}}$. We note that these Kähler forms are just those obtained by quotienting from the weighted Sasakian 3-sphere.

Lemma 2.5. Let $\boldsymbol{p}$ be admissible. Then quotient space of $\mu_{\eta_{0}}^{-1}(0) \approx S^{3} \times S^{3}$ by the $T^{2}(\boldsymbol{p})$ action is the orbifold $\mathbb{C P}\left(\bar{p}_{1}, \bar{p}_{2}\right) \times \mathbb{C P}\left(\bar{p}_{3}, \bar{p}_{4}\right)$. Moreover, the cohomology class in $H_{\text {orb }}^{2}\left(\mathbb{C P}\left(\bar{p}_{1}, \bar{p}_{2}\right) \times \mathbb{C P}\left(\bar{p}_{3}, \bar{p}_{4}\right), \mathbb{Z}\right)$ of this orbibundle is the class of the Kähler form $\omega_{\boldsymbol{p}}=l \omega_{\bar{p}_{1}, \bar{p}_{2}}+k \omega_{\bar{p}_{3}, \bar{p}_{4}}$. 
Proof. The $T^{2}(\boldsymbol{p})$ action on $S^{3} \times S^{3}$ splits as a weighted $S^{1}$ action on each factor. Setting $k=\operatorname{gcd}\left(p_{1}, p_{2}\right)$ and $l=\operatorname{gcd}\left(p_{3}, p_{4}\right)$, we see after reparametrizing that the quotient of the first factor is $\mathbb{C P}\left(\bar{p}_{1}, \bar{p}_{2}\right)$, and similarly for the second factor.

We have an exact sequence of groups

$$
0 \rightarrow T(\boldsymbol{p}) \rightarrow T^{2}(\boldsymbol{p}) \rightarrow S^{1}\left(R_{\boldsymbol{p}}\right) \rightarrow 0,
$$

where $S^{1}\left(R_{p}\right)$ is the circle generated by the Reeb vector field $R_{p}$. Thus, we have the commutative diagram

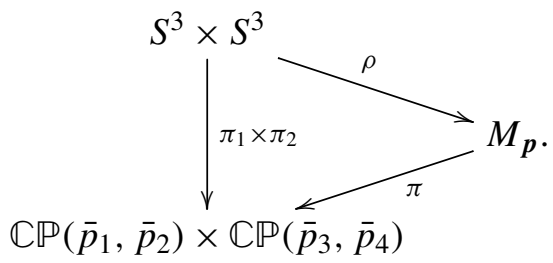

We want to determine the integral orbifold first Chern class (Euler class) of the $S^{1}$ orbibundle given by $\pi$. That is, we look for the class $a \alpha+b \beta \in H_{\text {orb }}^{2}\left(\mathbb{C P}\left(\bar{p}_{1}, \bar{p}_{2}\right) \times\right.$ $\left.\mathbb{C P}\left(\bar{p}_{3}, \bar{p}_{4}\right), \mathbb{Z}\right)$ which transcends to the zero class on $M_{p}$, where $\alpha$ and $\beta$ are primitive classes in each factor. (See [Boyer and Galicki 2008, Chapter 4] for a discussion of these orbifold classes.) For this we take $\alpha=\left[\omega_{\bar{p}_{1}, \bar{p}_{2}}\right]$, and $\beta=\left[\omega_{\bar{p}_{3}, \bar{p}_{4}}\right]$. Now according to the action (7) the circle wraps around $k$ times on the first factor and $l$ times with the reverse orientation on the second. So if we take the Kähler form to be

$$
\omega_{\boldsymbol{p}}=l \omega_{\bar{p}_{1}, \bar{p}_{2}}+k \omega_{\bar{p}_{3}, \bar{p}_{4}},
$$

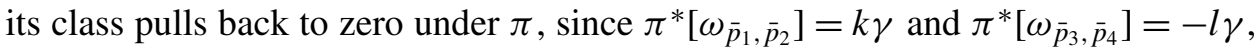
where $\gamma$ is a generator of $H_{2}\left(M_{p}, \mathbb{Z}\right) \approx \mathbb{Z}$.

The first Chern class and diffeomorphism types. In this subsection we relate the diffeomorphism type of our manifolds $M$ to the reduction parameters $\boldsymbol{p}$. We do this by giving a formula for the first Chern class of the contact bundle in terms of $\boldsymbol{p}$.

Theorem 2.6. $M_{\boldsymbol{p}}$ is diffeomorphic to $S^{2} \times S^{3}$ if $p_{1}+p_{2}-p_{3}-p_{4}$ is even, and diffeomorphic to $X_{\infty}$, the nontrivial $S^{3}$-bundle over $S^{2}$, if $p_{1}+p_{2}-p_{3}-p_{4}$ is odd.

Proof. We know from the reduction procedure and Lemma 2.2 that $M_{p}$ is simply connected and $\pi_{2}\left(M_{p}\right)=\mathbb{Z}$. So by the Barden-Smale classification of simply connected 5-manifolds $M_{p}$ is determined by its second Stiefel-Whitney class $w_{2}(M)$. Moreover, since $T M_{\boldsymbol{p}}$ splits as $\mathcal{D}_{\boldsymbol{p}}$ plus a trivial line bundle, $w_{2}(M)$ is the mod 2 reduction of $c_{1}(\mathcal{D})$. So the theorem will follow immediately from the following lemma. 
Lemma 2.7. The first Chern class of the contact bundle $\mathcal{D}_{p}=\operatorname{ker} \eta_{p}$ on $M_{p}$ is given by

$$
c_{1}\left(\mathcal{D}_{\boldsymbol{p}}\right)=\left(p_{1}+p_{2}-p_{3}-p_{4}\right) \gamma,
$$

where $\gamma$ is the positive generator of $H^{2}\left(M_{p}, \mathbb{Z}\right) \approx \mathbb{Z}$.

Proof. We begin by computing the orbifold first Chern class of

$$
\mathbb{C P}\left(\bar{p}_{1}, \bar{p}_{2}\right) \times \mathbb{C P}\left(\bar{p}_{3}, \bar{p}_{4}\right) .
$$

From [Boyer and Galicki 2008, Chapter 4] we see that $p^{*} c_{1}^{\text {orb }}$ is given by

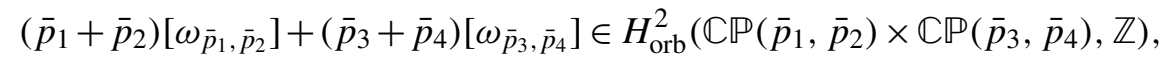

which pulls back to the basic first Chern class $c_{1}\left(\mathcal{F}_{R_{p}}\right)$ in the basic cohomology group $H_{B}^{2}\left(\mathcal{F}_{R_{p}}\right)$ under the natural projection $\pi: M_{\boldsymbol{p}} \rightarrow \mathbb{C P}\left(\bar{p}_{1}, \bar{p}_{2}\right) \times \mathbb{C P}\left(\bar{p}_{3}, \bar{p}_{4}\right)$ by the circle action of $R_{p}$. Now we have an exact sequence

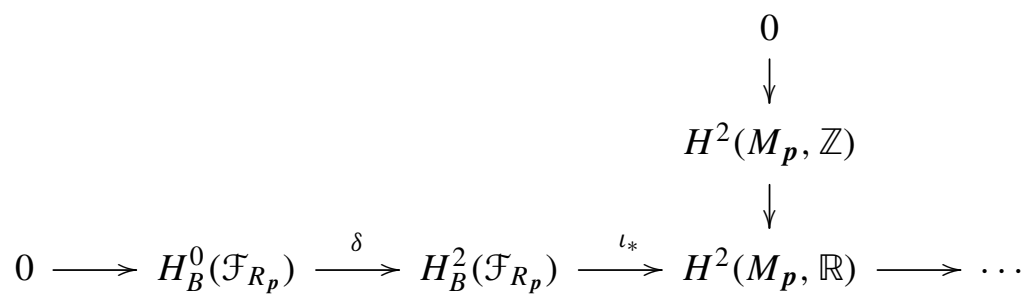

(see [Boyer and Galicki 2008, p. 245]), with $\iota_{*} c_{1}\left(\mathcal{F}_{R_{\boldsymbol{p}}}\right)=c_{1}\left(\mathcal{D}_{\boldsymbol{p}}\right)_{\mathbb{R}}$ and $\delta a=a\left[d \eta_{\boldsymbol{p}}\right]_{B}$. So $c_{1}\left(\mathcal{D}_{p}\right)_{\mathbb{R}}$ is $c_{1}\left(\mathcal{F}_{R_{p}}\right) \bmod \left[d \eta_{p}\right]_{B}$, where $\eta_{p}$ is the contact form on $M_{\boldsymbol{p}}$. Now since $\pi^{*} \omega_{\boldsymbol{p}}=d \eta_{\boldsymbol{p}}$, we know from the proof of Lemma 2.5 that $\pi^{*}\left[\omega_{\bar{p}_{1}, \bar{p}_{2}}\right]=k \gamma$

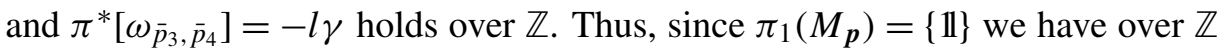

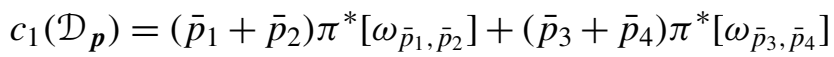

$$
\begin{aligned}
& =k\left(\bar{p}_{1}+\bar{p}_{2}\right) \gamma-l\left(\bar{p}_{3}+\bar{p}_{4}\right) \gamma=\left(p_{1}+p_{2}-p_{3}-p_{4}\right) \gamma .
\end{aligned}
$$

It is easy to see that the argument in [Lerman 2003b] for $S^{2} \times S^{3}$ can be generalized to the nontrivial bundle $X_{\infty}$ to give the following.

Proposition 2.8. As a complex vector bundle, $\mathcal{D}_{\boldsymbol{p}}$ is determined uniquely by $p_{1}+p_{2}-p_{3}-p_{4}$.

\section{Contact homology}

Here we apply pseudoholomorphic curve theory as briefly described on page 282 to the Morse-Bott formulation of contact homology. The study of pseudoholomorphic curves in symplectic manifolds was initiated in the seminal paper by Gromov [1985]. Since then, these objects have become a basic tool in understanding symplectic geometry and topology, perhaps most notably in the work of Floer, which is the main 
motivation behind symplectic field theory (SFT) and contact homology. The latter is a small part of the larger SFT of Eliashberg, Givental, and Hofer [Eliashberg et al. 2000]. The original idea, inspired by Floer homology, was to create a homology theory from the chain complex generated by closed orbits of the Reeb vector field.

Given a contact manifold $(M, \mathcal{D})$, we choose a contact form $\eta$ for $\mathcal{D}$, and an almost-complex structure $J$ on the symplectization of $M$ which extends the almostcomplex structure on $\mathcal{D}$ such that the Reeb vector field is the purely imaginary direction. For the moment, assume that periodic orbits of the Reeb vector field are isolated. This is a generic property of contact forms, and one can always find such a Reeb field for any contact structure. We consider the set of all closed Reeb orbits. We consider two orbits as different if they have different periods, even if they geometrically trace out the same set. Orbits with period one are often called simple orbits. We will consider the chain complex whose generators are periodic orbits of the Reeb vector field. The grading is given by the Robbin-Salamon index, which in the case of isolated orbits is the same as the well-known Conley-Zehnder index [Robbin and Salamon 1993]. The differential is given by an algebraic count of rigid $J$-holomorphic curves from a twice-punctured two-sphere into the symplectization which are asymptotically cylindrical over closed Reeb orbits, that is, they are curves for which there exist polar coordinates about each puncture, such that for sufficiently small radius the curve behaves like a cylinder over a closed Reeb orbit. If we look at such a curve in standard coordinates in the symplectization, we call punctures which correspond to limits as the real coordinate approaches positive infinity positive punctures; the others are called negative punctures [Hofer et al. 1996; Eliashberg et al. 2000].

Both the Robbin-Salamon indices arise from the Maslov index for a path of symplectic matrices. We compute the index of a closed Reeb orbit as follows: first, let us assume that $H_{1}(M, \mathbb{Z})=0$ and consider a closed Reeb orbit $\gamma$ together with an embedded Riemann surface $\Sigma \subset M$ such that $\partial \Sigma=\gamma$. To find the relevant path of symplectic matrices with which to compute the Maslov index, one then pulls back the contact bundle $\mathcal{D}$ to $\Sigma$, which then admits a trivialization, since it is a symplectic vector bundle over a Riemann surface with boundary. Then one considers the linearized Reeb flow about a Reeb orbit. This linearized flow gives the desired path of symplectic matrices. It is important to understand that in a contact manifold, these indices depend on the choice of capping disk used to trivialize $\mathcal{D}$. In particular, if the closed Reeb orbit $\gamma$ is contractible (which is always the case in this article), one trivializes $\mathcal{D}$ by choosing a capping disk $\Sigma$ of $\gamma$. If we consider another capping surface of the form $\Sigma^{\prime}=\Sigma \# S_{A}$ where $S_{A}$ represents a two-dimensional homology class $A$ in $M$, then the Conley-Zehnder (and Robbin-Salamon) index of the orbit computed with $\Sigma^{\prime}$ will differ from that computed using $\Sigma$ by twice the 
first Chern class of $\mathcal{D}$ evaluated on $A$, namely

$$
\mu_{\mathrm{CZ}}\left(\gamma ; \Sigma_{\gamma} \# S_{A}\right)=\mu_{\mathrm{CZ}}\left(\gamma ; \Sigma_{\gamma}\right)+2\left\langle c_{1}(\mathcal{D}), A\right\rangle .
$$

Thus, the grading depends on the choice of capping surface.

In order to address this dependence one considers the coefficients for contact homology to be elements in a Novikov ring as follows. Give $H_{2}(M, \mathbb{Z})$ a grading $|\cdot|$ by setting $|A|=-2\left\langle c_{1}(\mathcal{D}), A\right\rangle$ for any $A \in H_{2}(M, \mathbb{Z})$. Let $\mathcal{R}$ be a submodule of $H_{2}(M, \mathbb{Z})$ with zero grading. Then the Novikov ring is the graded group ring $\mathbb{Q}\left[H_{2}(M, \mathbb{Z}) / \mathcal{R}\right]$ whose elements are formal power series of the form $\sum_{i} q_{i} e^{A_{i}}$, where $q_{i} \in \mathbb{Q}$ and $A_{i} \in H_{2}(M, \mathbb{Z}) / \mathcal{R}$. Here as usual the notation $e^{A}$ is used to encode the multiplicative structure of a commutative ring with unit (see [McDuff and Salamon 2004, Chapter 11]).

There are some Reeb orbits for which the moduli space of holomorphic curves in $C(M)$ cannot be given a coherent orientation [Bourgeois and Mohnke 2004] these "bad" Reeb orbits must be discarded. Let $\gamma$ be a Reeb orbit with minimal period $T$, and $\gamma_{m}$ be a Reeb orbit that covers $\gamma$ with multiplicity $m$, so the period of $\gamma_{m}$ is $m T$. The bad orbits are those for which the parity of the even multiples $\left|\gamma_{2 m}\right|$ disagrees with the parity for the odd multiples $\left|\gamma_{2 m-1}\right|$. A Reeb orbit that is not bad is said to be good.

Now that we have a grading, under favorable circumstances we can define a graded chain complex $C_{*}$ generated by certain closed Reeb orbits with coefficients in the ring $\mathbb{Q}\left[H_{2}(M, \mathbb{Z}) / \mathcal{R}\right]$.

Definition 3.1. We define $C_{*}$ to be the graded chain complex freely generated by all good closed Reeb orbits with coefficients in the Novikov ring $\mathbb{Q}\left[H_{2}(M, \mathbb{Z}) / \mathcal{R}\right]$. By convention, we shift all degrees by $n-2$, where $2 n+1$ is the dimension of the contact manifold. The contact homology, denoted $H C(\mathcal{D})$, is the homology of the differential graded algebra $C_{*}$ with differential given by (18).

The differential $\partial$ of this chain complex is given by an algebraic count of pseudoholomorphic curves in the symplectization $C(M)$ of $M$ which come in one-dimensional families. Explicitly, for $\gamma$ a good closed orbit of the Reeb vector field, $M$ simply connected, and $A$ a two-dimensional homology class, the differential is given by the formula

$$
\partial \gamma=\sum_{A \in H_{2}(M, \mathbb{Z})} \sum_{\gamma^{\prime}} \frac{1}{\kappa_{\gamma}} n_{\gamma, \gamma^{\prime}, A} e^{\bar{A}} \gamma^{\prime},
$$

where $\bar{A}$ denotes the image in $H_{2}(M, \mathbb{Z}) / \mathcal{R}$ of the homology class $A, \kappa_{\gamma}$ is the multiplicity of the Reeb orbit $\gamma$, and $n_{\gamma, \gamma^{\prime}}$ is the algebraic count of elements in the moduli space $\mathcal{M}^{A}\left(\gamma, \gamma^{\prime}\right)$ of $J$-holomorphic curves into the symplectization of $M$ 
which are asymptotically cylindrical over the closed Reeb orbits $\gamma, \gamma^{\prime}$ representing the homology class $A$.

Note that $n_{\gamma, \gamma^{\prime}, A}$ is nonzero only if the dimension of this moduli space is 1 . This indeed gives a reasonable homology. The proofs that $\partial^{2}=0$ and that the homology does not depend on choices of a contact form or an almost-complex structure come from analysis of the boundary of moduli spaces of rigid curves and are discussed in [Eliashberg et al. 2000]. These results, in general, depend on abstract transversality results for the $\bar{\partial}_{J}$ operator. We will make the standing assumption that such transversality can be achieved, either by abstract perturbations or by the amenable geometry of the situation at hand. The signs which appear in the algebraic count depend on coherent orientations of the moduli space are explained in [Bourgeois and Mohnke 2004].

Remark 3.2. Due to the lack of compactness of moduli spaces of pseudoholomorphic cylinders, $\partial^{2}$ is not always zero. If it is, then the homology is often called cylindrical contact homology. Indeed, the boundary of the compactification of this space can, in general, contain curves with more than two punctures. However, we can instead consider the supercommutative algebra generated by periodic orbits. This means that instead of counting only cylinders, we now count curves with an arbitrary number of negative punctures. In this paper, it suffices to count cylinders.

Morse-Bott contact homology. In the above constructions we needed to make an assumption that the closed Reeb orbits are isolated in order to get a good index, that is, we have to assume that the Poincaré return map constructed about any periodic Reeb orbit has no eigenvalue equal to 1 . This condition is generic; however, many natural contact forms, especially those which arise from circle orbibundles, are as far from generic as possible. In order to calculate contact homology for such manifolds one must make some sort of perturbation. It is only in very nice situations that this is not extremely difficult. The Morse-Bott version [Eliashberg et al. 2000; Bourgeois 2002; 2003] allows us to use the symmetries of nice contact structures and symmetric almost-complex structures, by exploiting rather than excluding nonisolated orbits. This is accomplished by considering Morse theory on the quotient space, and relating critical points and gradient trajectories of a Morse function to pseudoholomorphic curves in the symplectization of the contact manifold. Since toric contact manifolds of Reeb type are always total spaces of circle orbibundles admitting Hamiltonian actions of tori and they admit nice Morse functions, the Morse-Bott formalism works quite well for us. We follow a combination of [Eliashberg et al. 2000] and [Bourgeois 2002] in what follows, applying the Morse-Bott setup to our special case.

Let $(M, \mathcal{D})$ be a contact manifold with contact form $\eta$, and consider the action 
functional $\mathfrak{A}: C^{\infty}\left(S^{1} ; M\right) \rightarrow \mathbb{R}$, defined by

$$
\mathfrak{A}(\gamma)=\int_{\gamma} \eta
$$

The critical points of $\mathfrak{A}$ are the closed orbits of the Reeb vector field of $\eta$. The action spectrum is defined to be

$$
\sigma(\eta)=\{r \in \mathbb{R} \mid r=\mathfrak{A}(\gamma)\}
$$

for $\gamma$ a periodic orbit of the Reeb vector field. Given $T \in \sigma(\eta)$, define

$$
N_{T}=\left\{p \in M \mid \phi_{p}^{T}=p\right\}, \quad S_{T}=N_{T} / S^{1},
$$

where $S^{1}$ acts on $M$ via the Reeb flow. Then $S_{T}$ is called the orbit space for period $T$.

When $M$ is the total space of an $S^{1}$-orbibundle the orbit spaces are precisely the orbifold strata. This is a special case of a contact form being of Morse-Bott type:

Definition 3.3. A contact form, $\eta$ is said to be of Morse-Bott type if

(i) The action spectrum $\sigma(\eta)$ is discrete.

(ii) The sets $N_{T}$ are closed submanifolds of $\mathbf{M}$, such that the rank of $\left.d \eta\right|_{N_{T}}$ is locally constant and

$$
T_{p}\left(N_{T}\right)=\operatorname{ker}\left(d \phi_{T}-I\right) .
$$

Remark 3.4. These conditions are the Morse-Bott analogues for the functional on the loop space of $M$.

Rather than set up Morse-Bott contact homology in full generality, let us do this for the special case of an $S^{1}$-orbibundle. In this case the contact form is of Morse-Bott type [Bourgeois 2002]. Let $T_{1}, \ldots, T_{m}$ be all possible simple periods for closed Reeb orbits. Let $\phi_{x}^{t}$ denote the flow of the Reeb vector field. Let

$$
N_{T_{j}}=\left\{x \in M \mid \phi_{x}^{T_{j}}=x\right\}, \quad S_{T_{j}}=N_{T_{j}} / S^{1} .
$$

For each $j$, we choose a Morse function $f_{j}$ on $S_{T_{j}}$ and, using appropriate bump functions, build a Morse function $f$ on all of $M$ which descends under the quotient by the Reeb action to each orbit space. Now, we perturb $\eta$ by

$$
\eta_{f}=(1+\epsilon f) \eta
$$

For almost all $\epsilon$, the closed Reeb orbits of $\eta_{f}$ are isolated, and, for bounded action, they correspond to critical points of $f$. Note that the Reeb orbits of $\eta$ within each stratum all have the same Robbin-Salamon index. The following formula [Cieliebak 
et al. 1996; Bourgeois 2002] computes the Conley-Zehnder index of $\eta_{f}$ in terms of the Robbin-Salamon index of any Reeb orbit in a particular orbit space:

$$
|\gamma|=\mu_{\mathrm{CZ}}(\gamma)=\mu_{\mathrm{RS}}\left(\gamma_{T}\right)-\frac{1}{2} \operatorname{dim}\left(S_{T_{j}}\right)+\operatorname{ind}_{p}\left(f_{j}\right) .
$$

We now have a contact form with isolated closed Reeb orbits. Since these orbits correspond to critical points of a Morse function, we may think of the generators of contact homology either as isolated orbits, or as critical points of a Morse function on each orbit space. This gives the generators and their gradings for the chain complex (or differential graded algebra) of Morse-Bott contact homology when we add, by convention, the degree shift $n-2$, which is 0 in the five-dimensional case. We now describe the differential $\partial$; however, we shall be very brief, as the differential vanishes identically in our case, as proven in Theorem 3.9.

Consider orbit spaces $S_{T_{1}}$ and $S_{T_{2}}$, for $T_{1}, T_{2} \in \sigma(\eta)$. Now define the moduli space $\mathcal{M}_{J}\left(S_{T_{1}}, S_{T_{2}}\right)$ to be the space of pseudoholomorphic curves (with respect to $J$ ) into $C(M)$ with one positive and one negative puncture which are asymptotically cylindrical over a closed Reeb orbit in $S_{T_{1}}$ near the positive puncture, and to $S_{T_{2}}$ near the negative puncture. We require, moreover, that these curves have finite, nonzero area.

In the case at hand the differential splits into two pieces:

$$
\partial p=\partial_{\mathrm{MSW}} p+d_{\mathrm{CH}} S,
$$

where $\partial_{\mathrm{MSW}}$ is the differential on the Morse-Smale-Witten complex determined by our choice of Morse function on the orbit space $S$ containing $p$, and roughly speaking $d_{\mathrm{CH}} S$ gives a count of rigid pseudoholomorphic curves in $\mathcal{M}_{J}\left(S, S^{\prime}\right)$. Here $S^{\prime}$ is some orbit space with action less than that of $S$. The count is over all $S^{\prime}$ such that the dimension of $\mathcal{M}_{J}\left(S, S^{\prime}\right)$ is equal to 1 . We refer to [Bourgeois 2002; Pati 2009] for more details. The following proposition shows the vanishing of $d_{\mathrm{CH}}$ for orbibundles.

Proposition 3.5. When $M$ is the total space of an orbibundle of a symplectic orbifold, then there are no rigid holomorphic curves into the symplectization of $M$.

Proof. There is an effective $\mathbb{R}$-action, as well as that of a circle on the moduli spaces; hence, these spaces have dimension at least two. So they can never be rigid.

Remark 3.6 (on transversality). Though in some special cases we can use the nice properties of toric manifolds to determine regularity of the moduli spaces of curves defined above, the proof of invariance of contact homology as well as the proof that the Morse-Bott complex actually computes the homology of the perturbed complex requires the use of abstract perturbations of the $\bar{\partial}_{J}$ operator. We believe that the results of Hofer, Wysocki, and Zehnder's polyfold theory will provide a good framework for this problem; however, we make it a standing assumption that 
there exists an abstract perturbation of the $\bar{\partial}_{J}$ operator which makes its linearization surjective. Proposition 3.5 requires no transversality result for the $\bar{\partial}_{J}$ operator since we can get at least these two dimensions without any appeal to abstract Fredholm theory. This does not make the transversality problem go away, however, since it is still needed in proofs of invariance, and independence of choices. Moreover, when one wishes to analyze higher-dimensional moduli spaces by adding marked points, one needs the relevant dimension formulae to hold, although this can be handled in many cases using the fact that $J$ can be chosen to be integrable in these toric situations. We should also mention that, even without the transversality assumption mentioned here, we can obtain a weaker version of invariance, as we shall see later.

Contact homology for toric contact 5-manifolds. Let us consider the differential graded algebra discussed above. We start with the set of critical points of a Morse function as picked earlier. Since we are working with toric manifolds of Reeb type in dimension 5 we actually know that the fixed points of the $T^{3}$-action are isolated, hence the norm squared of the symplectic moment map on $z$ is a perfect Morse function.

We are interested in the orbit structure of the $T^{3}(\boldsymbol{p})$ action on $M_{\boldsymbol{p}}$.

Lemma 3.7. Consider the toric contact structure $\mathcal{D}_{p}$ on $M_{p}$, an $S^{3}$-bundle over $S^{2}$. There are exactly four one-dimensional simple closed orbits under the action of $T^{3}(\boldsymbol{p})$. Moreover, these four orbits are Reeb orbits for all Reeb fields in the Sasaki cone $\mathrm{t}_{3}^{+}(\boldsymbol{p})$, as well as for a Reeb vector field in $\mathfrak{c o n}\left(M_{p}, \eta_{p}\right)$ that is arbitrarily close to one in the Sasaki cone. Moreover, for a generic such Reeb vector field these are the only closed orbits.

Proof. We have an exact sequence of groups,

$$
\{0\} \rightarrow T(\boldsymbol{p}) \rightarrow T^{4} \rightarrow T^{3}(\boldsymbol{p}) \rightarrow\{0\},
$$

and we consider the action of $T^{3}(\boldsymbol{p})$ on the level set given by (13) thought of as $T^{4} / T(\boldsymbol{p})$. If $z_{1} \neq 0$, then we can choose $\theta=\theta_{1}$ of the standard $T^{4}$ angles. The remaining $T^{3}$ orbit will be one-dimensional only if $z_{2}=0$ and one of $z_{3}$ or $z_{4}$ is zero. This gives two closed $S^{1}$-orbits. On the other hand if $z_{1}=0$, then we must have $z_{2} \neq 0$, so we choose $\theta=\theta_{2}$, and as above this gives exactly the two close orbits with either $z_{3}$ or $z_{4}$ vanishing. Clearly, any Reeb vector field in $t_{3}^{+}$leaves these Reeb orbits invariant, and since every Reeb vector field in the Sasaki cone is arbitrarily close to a quasiregular one, the last statement follows from a result of Bourgeois [2002].

Let $\mathfrak{t}_{2}(\boldsymbol{p})$ denote the Lie algebra of $T^{2}(\boldsymbol{p})$. It is generated by the two vector fields $L_{\boldsymbol{p}}$ and $R_{\boldsymbol{p}}$. We have an exact sequence of Lie algebras

$$
\{0\} \rightarrow \mathfrak{t}_{2}(\boldsymbol{p}) \rightarrow \mathfrak{t}_{4} \stackrel{\rho}{\rightarrow} \mathfrak{g}_{2}(\boldsymbol{p}) \rightarrow\{0\},
$$


where $\mathfrak{g}_{2}(\boldsymbol{p})$ is generated by the vector fields $\bar{H}_{1}=\rho\left(H_{1}\right)$ and $\bar{H}_{3}=\rho\left(H_{3}\right)$. We have a toric symplectic orbifold

$$
\left(\mathbb{C P}\left(\bar{p}_{1}, \bar{p}_{2}\right) \times \mathbb{C P}\left(\bar{p}_{3}, \bar{p}_{4}\right), \omega_{\boldsymbol{p}}\right),
$$

where the symplectic form is given by (15), and the torus $\mathfrak{G}_{2}(\boldsymbol{p})$ is generated by the Lie algebra $\mathfrak{g}_{2}(\boldsymbol{p})$. The moment map

$$
\mu_{2}: \mathbb{C P}\left(\bar{p}_{1}, \bar{p}_{2}\right) \times \mathbb{C P}\left(\bar{p}_{3}, \bar{p}_{4}\right) \rightarrow \mathfrak{g}_{2}(\boldsymbol{p})^{*}
$$

is given by $\mu_{2}(z)=\left(\left|z_{1}\right|^{2},\left|z_{3}\right|^{2}\right)$.

Proposition 3.8. The function $f=\left|\mu_{2}\right|^{2}$ is a perfect Morse function on the quotient $M_{p} / S^{1} \approx \mathbb{C P}\left(\bar{p}_{1}, \bar{p}_{2}\right) \times \mathbb{C P}\left(\bar{p}_{3}, \bar{p}_{4}\right)$ whose critical points are precisely the four Reeb orbits of Lemma 3.7.

Proof. Since the critical points are isolated $f$ is a Morse function, and Morse-Bott functions that are the norm squared of a moment map are perfect [Lerman and Tolman 1997]. It is easy to check directly (see also [Kirwan 1984, Lemma 3.1]), using the relations

$$
H_{1} \equiv a H_{2} \quad \bmod \mathfrak{g}_{2}(\boldsymbol{p}), \quad H_{3} \equiv b H_{4} \quad \bmod \mathfrak{g}_{2}(\boldsymbol{p}),
$$

for some $a, b \in \mathbb{R}$, that $f$ has precisely the four critical points

$$
[1,0] \times[1,0], \quad[1,0] \times[0,1], \quad[0,1] \times[1,0], \quad[0,1] \times[0,1],
$$

and these correspond to the four Reeb orbits of Lemma 3.7.

Theorem 3.9. In the case of circle reductions in dimension five, which have four Reeb orbits fixed by the $T^{3}$-action, the differential in Morse-Bott contact homology vanishes. Moreover, the elements of contact homology $H C(\mathcal{D})$ are given by the good Reeb orbits including multiplicity. More precisely, it is given by the homology groups of each stratum of its orbit space. The degree of each generator is given by (21).

Proof. By Proposition 3.5 there are no rigid holomorphic curves. So $d_{\mathrm{CH}}$ vanishes. But also by Proposition $3.8|\mu|_{2}^{2}$ is a perfect $S^{1}$-invariant Morse function, and the

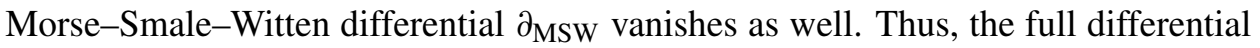
(22) vanishes. It then follows that the elements of $H C(\mathcal{D})$ are simply the chains of the complex $C_{*}$, that is, good closed Reeb orbits including multiplicity. For each period of the Reeb flow we get a different Reeb orbit, corresponding to some critical point of $f$. Since $f$ is perfect, these critical points correspond not just to chains but to actual homology classes. The statement about the grading follows from (21). 
The next proposition, though not a general proof of invariance of contact homology, does tell us that we do get an invariant in the world of $S^{1}$-orbibundles, whose bases admit a perfect Morse function.

Proposition 3.10. Let $M$ be a quasiregular contact manifold, such that its quotient by the Reeb vector field is a symplectic orbifold which admits a perfect Morse function. Then if $M^{\prime}$ is contactomorphic to $M$, is quasiregular, and the quotient by its Reeb vector field is also a symplectic orbifold which admits a perfect Morse function, then the two contact homology algebras are isomorphic.

Proof. The conditions on $M, M^{\prime}$, and their bases ensure that all parts of the differential vanish. Therefore we may construct a map between these two algebras as in [Eliashberg et al. 2000] counting rigid curves in a symplectic cobordism between $M$ and $M^{\prime}$. The main difficulty is in seeing that this map is a chain map. However, since the differentials vanish on both ends, the map is trivially a chain map, hence the two contact homology algebras are isomorphic.

The next proposition gives an nonequivalence statement about toric contact manifolds of type $\left(p_{1}, p_{2}, l, l\right)$.

Proposition 3.11. Let $\left(p_{1}, p_{2}, l, l\right)$ and $\left(p_{1}^{\prime}, p_{2}^{\prime}, l^{\prime}, l^{\prime}\right)$ be two admissible 4-tuples. If $p_{1}+p_{2} \neq p_{1}^{\prime}+p_{2}^{\prime}$, then the corresponding contact manifolds cannot be contactomorphic.

The proof of Proposition 3.11 is essentially an index calculation in light of Theorem 3.9. Let us first collect some information about the contact structures in question in convenient coordinates. To compute the grading on contact homology it is useful to consider a special case of the join construction [Boyer et al. 2007]. Since we can view our toric sphere bundles as quotients of $S^{3} \times S^{3}$ we have a convenient way to compute indices. This is of particular interest for strata of positive codimension, since the orbits in the codimension-zero stratum behave exactly as in the regular case. To define the join construction we start with two quasiregular contact manifolds, $M_{1}$ and $M_{2}$, with contact forms $\eta_{1}$ and $\eta_{2}$, and bases $z_{1}$ and $z_{2}$ with symplectic forms $\omega_{1}$ and $\omega_{2}$. Then the product $M_{1} \times M_{2}$ is a $T^{2}$-bundle over $z_{1} \times z_{2}$. We take the quotient of $M_{1} \times M_{2}$ by the action of the circle obtained by gluing together Reeb orbits on each piece, that is,

$$
(z, w) \mapsto\left(e^{i k_{1} \theta} z, e^{-i k_{2} \theta} w\right) .
$$

The admissibility conditions of Definition 2.4 are precisely the conditions that guarantee that the quotient by this action is smooth in which case it yields a new quasiregular contact manifold with base $z_{1} \times z_{2}$, contact form $\eta_{1}+\eta_{2}$, contact distribution given by $\mathcal{D}_{1} \oplus \mathcal{D}_{2}$, and Reeb vector field $R_{\eta_{1}}+R_{\eta_{2}}$. This contact structure is exactly the one coming from the principal circle bundle obtained by 
requiring that its curvature form is the pullback of the sum of the two symplectic forms on each base space. We obtain new Reeb orbits as equivalence classes of pairs of Reeb orbits, one from each of $M_{1}$ and $M_{2}$. When $k_{1}$ and $k_{2}$ are different from 1, we have a similar contact manifold, except the curvature is given by pulling back $k_{1}$ and $k_{2}$ multiples of the symplectic forms, namely $d \alpha=\pi^{*}\left(k_{2} \omega_{1}+k_{1} \omega_{2}\right)$. In this case Reeb orbits in the new total space will correspond to pairs, one wrapping $k_{1}$ and the other wrapping $k_{2}$ times (in addition to the multiplicity of the orbit as a Reeb orbit in one of the three-spheres). In the following, $M_{1}$ and $M_{2}$ are both standard three-spheres. Index calculations on three-dimensional spheres are standard; however, we present the details in Lemma 3.12 for completeness and also to illustrate the inherent role of the orbifold structure.

Let us consider the contact structure on the quotient of the product of two standard weighted three-spheres with weights $p_{1}, p_{2}, p_{3}$, and $p_{4}$. As before we take $k_{1}=\operatorname{gcd}\left(p_{1}, p_{2}\right), k_{2}=\operatorname{gcd}\left(p_{3}, p_{4}\right)$, and $\bar{p}_{i}=p_{i} / k_{1}$ for $i=1,2$, and $\bar{p}_{j}=p_{j} / k_{2}$ for $j=3,4$. We view this as a product of hypersurfaces in $\mathbb{C}^{4}$ with coordinates $\left(z_{1}, z_{2}, z_{3}, z_{4}\right), z_{j}=x_{j}+i y_{j}$, subject to the action (25). This manifold is the total space of an orbibundle over an orbifold $S^{2} \times S^{2}$ with orbifold singularities at the products of the north and south poles, and for the products of the north and south poles with copies of $S^{2}$. These singularities correspond to setting one or two of the $z_{j}$ to 0 . The Reeb vector field is given by

$$
p_{1} y_{1} \partial_{x_{1}}-x_{1} p_{1} \partial_{y_{1}}+p_{2} y_{2} \partial_{x_{2}}-p_{2} x_{2} \partial_{y_{4}}+p_{3} y_{3} \partial_{x_{3}}-x_{3} p_{3} \partial_{y_{3}}+p_{4} y_{4} \partial_{x_{4}}-p_{4} x_{4} \partial_{y_{4}}
$$

and the contact distribution is given by the span of the vectors

$$
\begin{aligned}
& -\frac{1}{p_{1}} x_{2} \partial_{x_{1}}+\frac{1}{p_{1}} y_{2} \partial_{y_{1}}+\frac{1}{p_{2}} x_{1} \partial_{x_{2}}-\frac{1}{p_{2}} y_{1} \partial_{y_{2}}, \\
& -\frac{1}{p_{1}} y_{2} \partial_{x_{1}}-\frac{1}{p_{1}} x_{2} \partial_{y_{1}}+\frac{1}{p_{2}} y_{1} \partial_{x_{2}}+\frac{1}{p_{2}} x_{1} \partial_{y_{2}}, \\
& -\frac{1}{p_{3}} x_{4} \partial_{x_{3}}+\frac{1}{p_{3}} y_{4} \partial_{y_{3}}+\frac{1}{p_{4}} x_{3} \partial_{x_{4}}-\frac{1}{p_{4}} y_{3} \partial_{y_{4}}, \\
& -\frac{1}{p_{3}} y_{4} \partial_{x_{3}}-\frac{1}{p_{3}} x_{4} \partial_{y_{3}}+\frac{1}{p_{4}} y_{3} \partial_{x_{4}}+\frac{1}{p_{4}} x_{3} \partial_{y_{4}} .
\end{aligned}
$$

In the following we restrict ourselves to the case where $p_{3}=p_{4}=k_{2}$. To get our hands on an orbit in the quotient, we must, for each time around the fiber, pick an appropriate circle out of the fiber of the torus bundle. It is easy to see that the equivalence relation gives us a circle obtained by wrapping around the first circle $k_{2}$ times and around the second circle $k_{1}$ times. Let us now parametrize the fiber. We may choose a coordinate for a Reeb orbit by

$$
\gamma(t)=\left(0, \cos \left(k_{2} \bar{p}_{2} t\right)+i \sin \left(k_{2} \bar{p}_{2} t\right), 0, \cos \left(p_{2} t\right)+i \sin \left(p_{2} t\right)\right) .
$$


Now when $t=1 / \bar{p}_{2}$ we have wrapped around the first orbit $k_{2}$-times and the second $k_{1}$ times. Here the action is $1 / p_{2}$. This is the smallest action since we have assumed that $p_{2}>p_{1}$. What about when the first orbit wraps around more than once in $S^{3}$ ? Let us see how to look at such an orbit. This corresponds to taking

$$
\gamma(t)=\left(\cos \left(k_{2} \bar{p}_{1} t\right)+i \sin \left(k_{2} \bar{p}_{1} t\right), 0,0, \cos \left(\frac{p_{1}}{m} t\right)+i \sin \left(\frac{p_{1}}{m} t\right)\right),
$$

where $m$ is the multiplicity. Now when $t=m / p_{1}$, we wrap around the first orbit $m k_{2}$ times and the second $k_{1}$ times. As long as $m<\min \left\{k_{2}, p_{1}\right\}$ we do not enter a higher-dimensional orbit space. Similar considerations remain true for $z_{1}=0$. Let us compute the Robbin-Salamon index of the orbits (30).

Lemma 3.12. The Robbin-Salamon indices of the orbits in (30) of multiplicity $m$ are given by

$$
2 k_{1} m+2\left\lfloor m p_{2} / p_{1}\right\rfloor-1, \quad 2 k_{1} m+2\left\lfloor m p_{1} / p_{2}\right\rfloor-1 .
$$

Proof. To do this we must choose a disk $D$ with boundary $\gamma$. Such a disk can be written explicitly. We begin by producing a disk in $S^{3} \times S^{3}$ :

$$
\left(\cos (\theta), \sin (\theta) e^{2 \pi i k_{2} \bar{p}_{2} t}, \cos (\theta), \sin (\theta) e^{2 \pi i p_{2} t / m}\right) .
$$

The above disk clearly has boundary $\gamma$ (the boundary occurs when $\theta=\pi / 2$ ) and we have $\theta \in[0, \pi / 2]$. To pull back the contact distribution we plug the coordinates into (26)-(29):

$$
\begin{aligned}
& -\frac{1}{p_{1}} \sin (\theta) \cos \left(2 \pi i k_{2} \bar{p}_{2} t\right) \partial_{x_{1}}+\frac{1}{p_{1}} \sin (\theta) \sin \left(2 \pi i k_{2} \bar{p}_{2} t\right) \partial_{y_{1}}+\frac{1}{p_{2}} \cos (\theta) \partial_{x_{2}}, \\
& -\frac{1}{p_{1}} \sin (\theta) \sin \left(2 \pi i k_{2} \bar{p}_{2} t\right) \partial_{x_{1}}-\frac{1}{p_{1}} \sin (\theta) \cos \left(2 \pi i k_{2} \bar{p}_{2} t\right) \partial_{y_{1}}+\frac{1}{p_{2}} \cos (\theta) \partial_{y_{2}} \\
& -\frac{1}{p_{3}} \sin (\theta) \cos \left(2 \pi i \frac{\bar{p}_{2} t}{m}\right) \partial_{x_{3}}+\frac{1}{p_{3}} \sin (\theta) \sin \left(2 \pi i \frac{\bar{p}_{2} t}{m}\right) \partial_{y_{3}}+\frac{1}{p_{4}} \cos (\theta) \partial_{x_{4}} \\
& -\frac{1}{p_{3}} \sin (\theta) \sin \left(2 \pi i \frac{\bar{p}_{2} t}{m}\right) \partial_{x_{3}}-\frac{1}{p_{3}} \sin (\theta) \cos \left(2 \pi i \frac{\bar{p}_{2} t}{m}\right) \partial_{y_{3}}+\frac{1}{p_{4}} \cos (\theta) \partial_{y_{4}} .
\end{aligned}
$$

When $\theta=\pi / 2$, these four vectors become

$$
\begin{aligned}
& \frac{1}{p_{1}}\left(-\cos \left(2 \pi i k_{2} \bar{p}_{2} t\right) \partial_{x_{1}}+\sin \left(2 \pi i k_{2} \bar{p}_{2} t\right) \partial_{y_{1}}\right), \\
& \frac{1}{p_{1}}\left(-\sin \left(2 \pi i k_{2} \bar{p}_{2} t\right) \partial_{x_{1}}-\cos \left(2 \pi i k_{2} \bar{p}_{2} t\right) \partial_{y_{1}}\right), \\
& \frac{1}{p_{3}}\left(-\cos \left(2 \pi i \frac{p_{2} t}{m}\right) \partial_{x_{3}}+\sin \left(2 \pi i \frac{p_{2} t}{m}\right) \partial_{y_{3}}\right), \\
& \frac{1}{p_{3}}\left(-\sin \left(2 \pi i \frac{p_{2} t}{m}\right) \partial_{x_{3}}-\cos \left(2 \pi i \frac{p_{2} t}{m}\right) \partial_{y_{3}}\right) .
\end{aligned}
$$


Disks for the other orbits mapping into branch divisors have a similar expression. The key point is that we only see vectors corresponding to the coordinates which have been set to zero. Now we can easily compute the Robbin-Salamon index of these orbits. Recall that given a path of symplectic matrices, $\Phi(t)$, a number $t$ is called a crossing if $\Phi(t)$ has an eigenvalue equal to 1 . To compute the RobbinSalamon index of a path of symplectic matrices on $[0, T]$ one computes

$$
\frac{1}{2} \text { signature }(\Gamma(0))+\sum_{\substack{\text { crossings } t \\ t \neq 0, T}} \text { signature }(\Gamma(t))+\frac{1}{2} \text { signature }(\Gamma(T)) .
$$

Here the crossing form is

$$
\varnothing(\dot{\Phi}(t) v, v)
$$

restricted to the subspace on which $\Phi$ has eigenvalues equal to 1 . In this case at each crossing the crossing form is just $\varnothing\left(v, J_{0} v\right)$, so this gives signature 2 on each two-dimensional subspace consisting of eigenvectors with eigenvalue 1 . At crossings the vectors above spanning $\mathcal{D}$ above become $-\left(1 / p_{1}\right) \partial x_{1},-\left(1 / p_{1}\right) \partial y_{1}$, $-(1 / l) \partial x_{3}$, and $-(1 / l) \partial y_{3}$.

Recall that the linearized Reeb flow is of the form

$$
\left[\begin{array}{cccc}
e^{2 \pi i p_{1} t} & 0 & 0 & 0 \\
0 & e^{2 \pi i p_{2} t} & 0 & 0 \\
0 & 0 & e^{2 \pi i k_{2} t} & 0 \\
0 & 0 & 0 & e^{2 \pi i k_{2} t}
\end{array}\right],
$$

and each complex block of the matrix looks like

$$
\left[\begin{array}{cc}
\cos \left(2 \pi p_{j} t\right) & -\sin \left(2 \pi p_{j} t\right) \\
\sin \left(2 \pi p_{j} t\right) & \cos \left(2 \pi p_{j} t\right)
\end{array}\right] .
$$

The time derivative of each block looks like

$$
\left[\begin{array}{cc}
-2 \pi p_{j} \sin \left(2 \pi p_{j} t\right) & -2 \pi p_{j} \cos \left(2 \pi p_{j} t\right) \\
2 \pi p_{j} \cos \left(2 \pi p_{j} t\right) & -2 \pi p_{j} \sin \left(2 \pi p_{j} t\right)
\end{array}\right] .
$$

At crossings these blocks become

$$
\left[\begin{array}{cc}
0 & -2 \pi p_{j} \\
2 \pi p_{j} & 0
\end{array}\right]
$$

The crossings which have the first two vectors as 1-eigenvectors occur at integers multiples of $1 /\left(k_{2} p_{1}\right)$, and those for the second two occur at integer multiples of $k_{1} m / k_{2}$. As we saw above the flow splits into two parts, that corresponding to the first two coordinates and that corresponding to the second two. This means the second part, for multiplicity $m$, is $2 m k_{1}$. Now we add the normal part. For orbits 
of multiplicity $m$ we get contribution $1+2\left\lfloor m / p_{1}\right\rfloor$. Therefore for multiplicity $m$ these orbits have Robbin-Salamon index

$$
2 k_{1} m+2\left\lfloor m p_{2} / p_{1}\right\rfloor-1,
$$

and similarly, setting $p_{2}$ to zero, we obtain

$$
2 k_{1} m+2\left\lfloor m p_{1} / p_{2}\right\rfloor-1 .
$$

We shall label these orbits $\gamma_{m, i}$. With this information we can now prove Proposition 3.11.

Proof of Proposition 3.11. The goal here is to distinguish contact structures. We will show that given two 4-tuples as in Proposition 3.11 that the contact homology algebras cannot be isomorphic. We know that $\left(p_{2}-1\right) / p_{2}>\left(p_{1}-1\right) / p_{1}$; hence the index of $\gamma$ is $2\left(p_{1}-1\right)$. This tells us that we have $p_{1}-1+p_{2}-1$ orbits of index less than $2\left(p_{2}-1\right)$.

Theorem 3.9 gives us a complete picture of the contact homology of the manifolds given by admissible 4-tuples up to knowing the Robbin-Salamon indices. Let us spell this out in the case $\left(p_{1}, p_{2}, k_{2}, k_{2}\right)$. In this case there are essentially two different kinds of orbit spaces. We have two-dimensional orbit spaces which project to two-spheres in the base, and we have copies of the whole manifold. The two-dimensional orbits spaces consist of orbits having action $k_{2} m / p_{i}$ for $p_{i} \nmid m$. The four-dimensional orbit spaces consist of orbits of integer action. For each two-dimensional orbit space, $S_{k_{2} m / p_{i}}$, we obtain exactly two orbits contributing to contact homology with grading difference two. We denote these orbits $\hat{\gamma}_{m, i}$, and $\check{\gamma}_{m_{i}}$ corresponding to the maximum and minimum of the Morse function on $S_{k_{2} m / p_{i}}$. For such orbits with action less than 1 we have grading

$$
\begin{aligned}
& \left|\hat{\gamma}_{m, i}\right|=\mu_{\mathrm{RS}}\left(\gamma_{m, i}\right)+1 \\
& \left|\check{\gamma}_{m, i}\right|=\mu_{\mathrm{RS}}\left(\gamma_{m, i}\right)-1 .
\end{aligned}
$$

For each four-dimensional orbit space we have four generators for contact homology, again corresponding to critical points. We label these

$$
\hat{\gamma}_{m}, \check{\gamma}_{m}, \gamma_{m}^{s_{1}}, \gamma_{m}^{s_{2}}
$$

for the maximum, minimum, and two saddle points, respectively. With a choice of disk $D$ projecting to the spherical homology class $\Sigma \in H_{2}(Z, \mathbb{Q})$ we have

$$
\begin{aligned}
\left|\hat{\gamma}_{m}\right| & =\mu_{\mathrm{RS}}\left(\gamma_{m}, D\right)+2, & & \left|\check{\gamma}_{m}\right|=\mu_{\mathrm{RS}}\left(\gamma_{m}, D\right)-2, \\
\left|\gamma_{m}^{s_{1}}\right| & =\mu_{\mathrm{RS}}\left(\gamma_{m}, D\right), & \left|\gamma_{m}^{s_{2}}\right| & =\mu_{\mathrm{RS}}\left(\gamma_{m}, D\right) .
\end{aligned}
$$

In (38) $\mu_{\mathrm{RS}}\left(\gamma_{m}, D\right)=2 k_{2} m\left\langle c_{1}^{\text {orb }}(z),(\Sigma)\right\rangle$. Moreover, for two-dimensional orbit spaces with action greater than 1 , by the catenation property of the Robbin-Salamon 
index, we may decompose the orbit into a part with biggest possible integer action and a part with action smaller than 1 . We then add the indices of these two orbits to get the Robbin-Salamon index. Note that for these two-dimensional orbit spaces, the tangential part of the flow is a loop, but the normal does not complete a loop; this explains the appearance of the summand 1 in the above formulae. Note that, here, the Robbin-Salamon index is nondecreasing with respect to action. Thus we may count the number of orbits with index less than 1 . This will give a count of generators of contact homology of index less than $2\left(p_{1}+p_{2}+2\right)-2$. From the above discussion there are $p_{1}-1+p_{2}-1$ such orbits coming from lower-dimensional orbit spaces, and then one coming from $\check{g}_{1}$. This gives exactly $p_{1}+p_{2}-1$ orbits in degree less than $2\left(p_{1}+p_{2}+1\right)$. This proves Proposition 3.11.

Proposition 3.11 applies directly to the $Y^{p, q}$ manifolds. In this case the invariant is $2 p-1$; note that it does not depend on $q$.

As an application let us use the preceding discussion to distinguish contact structures on the toric contact 5-manifolds corresponding to the 4-tuple $(1,2 k-1$, $l, l)$ for positive integers $k$ and $l$ such that the tuple $(1,2 k-1, l, l)$ is admissible. Then we see that $c_{1}(\mathcal{D})=2 k-2 l$. Let us fix the first Chern class of the contact distribution and see what happens. We see then that we must have

$$
k=\frac{c_{1}(\mathcal{D})+2 l}{2} .
$$

Now using Proposition 3.11 we see that there are $2 k-1$ generators in contact homology of degree less than $4 k+2$.

A remark on the regular case. In the regular case the situation is somewhat simpler, but, on the other hand, there is less information available at first glance. In this case there is geometrically only one orbit space, $z$ itself. To get a handle on the contact homology let us look at the case $(k, k, k-c, k-c)$. This gives a regular contact manifold with $c_{1}(\mathcal{D})=2 c$ times a generator. We choose a basis $L_{1}, L_{2}$ of $H_{2}(Z, \mathbb{Z})$ so that $L_{1}=x S_{1}+y S_{2}$, and $L_{2}$ lifts to a class which evaluates to 0 under $\pi^{*} \emptyset$. Both $x$ and $y$ are chosen so that they give action 1 for a disk that projects to $L_{1}$. We define $x$ and $y$ as follows. Let $m$ be the smallest number so that $m k \equiv-1$ $\bmod c$. Then we define $x=(k m+1) / c$ and $y=x-m$. It is easy to see that $x$ and $y$ satisfy the above properties. With these choices the grading for contact homology for orbits of action $N$ is given by

$$
|\hat{\gamma}|=N(2 x+2 y)+2, \quad|\check{\gamma}|=N(2 x+2 y)-2, \quad\left|\gamma_{N}^{s_{j}}\right|=N(2 x+2 y) .
$$

In this picture, for $N=1, \check{\gamma}$ gives the smallest possible grading. By varying $k$, we obtain infinitely many distinct contact structures whose contact distribution has the same Chern class. 
Another way to distinguish contact structures. As described in [Eliashberg et al. $2000, \S 2.9 .2]$, there is another situation where symplectic field theory can be used to distinguish toric contact structures. The following theorem is a generalization to smooth orbifolds of [Eliashberg et al. 2000, Proposition 2.9.4]:

Theorem 3.13. Suppose we have two simply connected quasiregular toric contact manifolds of Reeb type in dimension five, such that each orbifold stratum is nonsingular in the sense that its underlying space is a smooth submanifold. Suppose that under the quotient of the Reeb action one of the base manifolds has an exceptional sphere while the other does not, then these two manifolds are not contactomorphic.

Proof. We show that there is an odd element in the contact homology algebra of one manifold specialized at a class which is not in the other for any specialization. We assume here that all of the weights of the torus action are greater than 1 for the manifold containing no exceptional spheres. As in [Eliashberg et al. 2000] the potential specialized to the Poincaré dual of an exceptional divisor will give the potential for a standard $S^{3}$; but then for a chain which lifts to the volume form for this 3-form there is always a holomorphic curve to kill it as a generator for homology specialized at this three-class. Hence this homology contains no odd elements. Let us consider first the case where the base is a manifold. We look at the manifold containing no exceptional sphere. We must compute the Gromov-Witten potential (see the Appendix for a brief description). Unfortunately it does not vanish, but for any 2 -classes the potential always vanishes. This is because the Gromov-Witten invariant, $\mathrm{GW}_{A, k}^{0}(\alpha, \ldots, \alpha)$, is not equal to 0 for a two-dimensional class $\alpha$ only if

$$
2 k=4+2 c_{1}(A)+2 k-6, \quad \text { i.e., } c_{1}(A)=1 .
$$

But the weights make this impossible. Thus all coefficients for such curves vanish, and the potential vanishes on $z$, hence on $M$. So for a three-class in the contact manifold obtained from integration over the fiber of a two-class, there is no holomorphic curve to kill it. Hence specialized at such a three-class we have an odd generator which does not exist in the presence of exceptional spheres. The orbifold case is similar. The computation for the Gromov-Witten potential on the manifold with the exceptional sphere follows from the divisor axiom. To see that the coefficients for the Gromov-Witten potential vanish in the case where there is no exceptional sphere we note the Gromov-Witten invariant is nonzero only if the first Chern class evaluated on $A$ is equal to one minus the degree shifting number of $\boldsymbol{x}$, which in the absence of exceptional spheres in the stratum in question is impossible.

Remark 3.14. Since the base is four dimensional the results of [Hofer et al. 1997] tell us that we can indeed use the dimension formula above for computation of the Gromov-Witten invariants for the manifold case. To adjust for orbifold structure we 
use extra point conditions since all strata in this case are actually smooth manifolds additionally endowed with orbifold structure.

\section{The contact equivalence problem}

It is the purpose of this section to prove the contact equivalence of certain toric contact structures that are inequivalent as toric contact structures. To clarify this we say that two toric contact structures $\mathcal{D}_{\boldsymbol{p}}$ and $\mathcal{D}_{\boldsymbol{p}^{\prime}}$ are equivalent as contact structures, but inequivalent as toric contact structures, if there is a contactomorphism $\varphi$ : $M_{p} \rightarrow M_{p^{\prime}}$ such that $\varphi_{*} \mathcal{D}_{\boldsymbol{p}}=\mathcal{D}_{\boldsymbol{p}^{\prime}}$, but there is no $T^{3}$-equivariant contactomorphism. Then their 3-tori correspond to distinct conjugacy classes of maximal tori in the contactomorphism group [Lerman 2003b; Boyer 2013]. We remark that Theorems 1 and 2 are direct consequences of Theorem 4.11.

A complete answer to the equivalence problem appears to be quite difficult so we restrict ourselves to certain special cases of contact structures that are Seifert $S^{1}$-bundles (orbibundles) over Hirzebruch surfaces which generally have a nontrivial orbifold structure. In this case we show that certain $T^{3}$ equivariantly inequivalent contact structures are actually $T^{2}$ equivariantly equivalent for some subgroup $T^{2} \subset T^{3}$.

Orbifold Hirzebruch surfaces. In this section we study a special class of toric contact structures on $S^{3}$ bundles over $S^{2}$ that can be realized as circle orbibundles over orbifold Hirzebruch surfaces. Since the reduction method gives all examples of such toric contact structures, it is important to make contact (no pun intended) with examples that are known in the literature. Here we shall always assume that the quadruple $\boldsymbol{p}$ is admissible.

When working with Hirzebruch surfaces, we often follow [Griffiths and Harris 1978] (but with slightly different notation) and represent $S_{n}$ as the projectivized bundle $S_{n}=\mathbb{P}(\mathcal{O}(n)+\mathcal{O}) \rightarrow \mathbb{C P}^{1}$ with fibers $L=\mathbb{C P}^{1}$ and sections $E$ and $F$ with self-intersection numbers $n$ and $-n$, respectively. The sections, which satisfy $E \cdot E=n, E \cdot L=1$, and $L \cdot L=0$, define divisors in $S_{n}$ and determine a basis for the Picard group $\operatorname{Pic}\left(S_{n}\right) \approx H^{2}\left(S_{n}, \mathbb{Z}\right) \approx \mathbb{Z}^{2}$. However, when working with symplectic forms it is convenient to use a basis which appears for all admissible complex structures. Thus, it is convenient to treat the even and odd Hirzebruch surfaces separately. The even Hirzebruch surfaces $S_{2 n}$ are diffeomorphic to $S^{2} \times S^{2}$, so we define $E_{0}=E-n L$. Then we have

$$
E_{0} \cdot E_{0}=(E-n L) \cdot(E-n L)=E \cdot E-2 n E \cdot L+L \cdot L=2 n-2 n=0 .
$$

In this case the Poincare duals $\alpha_{L}$ and $\alpha_{E_{0}}$ are the standard area forms for the two copies of $S^{2}$. Similarly, the odd Hirzebruch surfaces $S_{2 n+1}$ are diffeomorphic to $\mathbb{C P}^{2}$ blown up at a point which we denote by $\widetilde{\mathbb{C P}^{2}}$. In this case we define 
$E_{-1}=E-(n+1) L$ which gives $E_{-1} \cdot E_{-1}=-1$. So $E_{-1}$ is an exceptional divisor. Again the Poincaré duals $\alpha_{L}$ and $\alpha_{E_{-1}}$ represent the standard area forms on a fiber and exceptional divisor, respectively.

As mentioned previously the orbifolds that we encounter are of the form $(X, \Delta)$, where $X$ is a smooth algebraic variety and $\Delta$ is a branch divisor. Specifically we are interested in the orbifolds $\left(S_{n}, \Delta\right)$, where $\Delta=\sum_{i}\left(1-1 / m_{j}\right) D_{j}$ and $D_{i}$ are Weil divisors on $S_{n}$. We refer to the pair $\left(S_{n}, \Delta\right)$ as an orbifold Hirzebruch surface. We now wish to compute the orbifold canonical divisor in this situation. Since we are working with $\mathbb{Q}$-divisors, we can express the result in terms of $E_{0}$ even though it is an honest divisor only on even Hirzebruch surfaces.

Lemma 4.1. Let $\left(S_{n}, \Delta\right)$ be an orbifold Hirzebruch surface such that $E$ and $F$ are branch divisors, both with ramification index $m$. Then the orbifold canonical divisor of $\left(S_{n}, \Delta_{m}\right)$ is

$$
K_{\left(S_{n}, \Delta_{m}\right)}^{\text {orb }}=-\frac{2}{m} E-\frac{2 m-n}{m} L=-\frac{2}{m} E_{0}-2 L .
$$

Hence $\left(S_{n}, \Delta_{m}\right)$ is a log del Pezzo surface (Fano) if and only if $2 m>n$.

Proof. We know [Griffiths and Harris 1978, p. 519] that the canonical divisor $K_{S_{n}}$ of $S_{n}$ is given by $K_{S_{n}}=-2 E+(n-2) L=-2 E_{0}-2 L$, and the orbifold canonical divisor $K_{S_{n}, \Delta_{m}}^{\text {orb }}$ satisfies (see [Boyer and Galicki 2008, p. 127])

$$
K_{S_{n}, \Delta_{m}}^{\text {orb }}=K_{S_{n}}+\left(1-\frac{1}{m}\right)(E+F) .
$$

Now the divisor $E$ has self-intersection $n$, and the divisor $F$ has self-intersection $-n$, and since they both have intersection 1 with the fiber $L$, we have $(E+F)=2 E_{0}$. Putting this together gives the formula.

The orbifold $\left(S_{n}, \Delta_{m}\right)$ is log del Pezzo if and only if the orbifold anticanonical divisor $-K_{\left(S_{n}, \Delta_{m}\right)}^{\mathrm{orb}}$ is ample, and this happens if and only if $2 m>n$ by Nakai's criterion since $E$ and $L$ are effective.

Toric contact structures on $\boldsymbol{S}^{\mathbf{2}} \times \boldsymbol{S}^{\mathbf{3}}$. The toric contact structures we describe here are not the most general, but are obtained by setting $\boldsymbol{p}=(j, 2 k-j, l, l)$. That is, we consider contact structures of the form $\mathcal{D}_{j, 2 k-j, l, l}$ where the pair $(k, l)$ is fixed with $k \geq l$, and $j=1, \ldots, k$. Now since $\boldsymbol{p} \in \mathcal{A}$ we also have $\operatorname{gcd}(j, l)=\operatorname{gcd}(2 k-j, l)=1$. We denote the set of $j=1, \ldots, k$ such that $\boldsymbol{p}=(j, 2 k-j, l, l)$ is admissible by $\mathcal{J}_{\mathcal{A}}=$ $\mathcal{J}_{\mathcal{A}}(k, l)$. The first Chern class of this contact structure is $c_{1}\left(\mathcal{D}_{j, 2 k-j, l, l}\right)=2(k-l) \gamma$ where $\gamma$ is a generator of $H^{2}\left(M_{\boldsymbol{p}}, \mathbb{Z}\right) \approx \mathbb{Z}$. So in this case $M_{\boldsymbol{p}}$ is $S^{2} \times S^{3}$. The infinitesimal generator of the circle action is $L_{p}=j H_{1}+(2 k-j) H_{2}-l H_{3}-l H_{4}$. Note that this case includes the $Y^{p, q}$ as a special case, namely, $p=k=l$ and $q=k-j$. So $Y^{p, q}$ is $\mathcal{D}_{p-q, p+q, p, p}$ with $p>q$ and $\operatorname{gcd}(p, q)=1$. 
We want to find a suitable Reeb vector field in the Sasaki cone, so we try $R_{j, k, l}=(2 k-j) H_{1}+j H_{2}+l H_{3}+l H_{4}$ which clearly satisfies the positivity condition $\eta_{0}(R)>0$. The $T^{2}$ action generated by $L_{p}$ and $R_{j, k, l}$ is

$$
z \mapsto\left(e^{i((2 k-j) \phi+j \theta)} z_{1}, e^{i(j \phi+(2 k-j) \theta)} z_{2}, e^{i l(\phi-\theta)} z_{3}, e^{i l(\phi-\theta)} z_{4}\right) .
$$

Making the substitutions $\psi=\phi-\theta$ and $\chi=j \psi+2 k \theta$ gives the action

$$
z \mapsto\left(e^{i(2(k-j) \psi+\chi)} z_{1}, e^{i \chi} z_{2}, e^{i l \psi} z_{3}, e^{i l \psi} z_{4}\right) .
$$

We define $g_{j}=\operatorname{gcd}(l, 2(k-j))$ and write $2(k-j)=n_{j} g_{j}$ and $l=m_{j} g_{j}$. Then $\operatorname{gcd}\left(m_{j}, n_{j}\right)=1$.

Theorem 4.2. Consider the contact manifold $\left(S^{2} \times S^{3}, \mathcal{D}_{j, 2 k-j, l, l}\right)$ where $1 \leq j \leq k$ satisfies $\operatorname{gcd}(j, l)=\operatorname{gcd}(2 k-j, l)=1$. Then we have

(1) The quotient space by the circle action generated by the Reeb vector field $R=(2 k-j) H_{1}+j H_{2}+l H_{3}+l H_{4}$ is the Kähler orbifold $\left(S_{n_{j}}, \Delta ; \omega_{k, l, j}\right)$ where $S_{n_{j}}$ is a Hirzebruch surface, $\Delta$ is the branch divisor,

$$
\Delta=\left(1-\frac{1}{m_{j}}\right)(E+F),
$$

and $\omega_{k, l, j}$ is an orbifold symplectic form satisfying $\pi^{*} \omega_{k, l, j}=d \eta_{k, l, j}$, where $\eta_{k, l, j}$ is the contact 1 -form representing $\mathcal{D}_{j, 2 k-j, l, l}$ whose Reeb vector field is $R$.

(2) The orbifold structure is trivial $(\Delta=\varnothing)$ if and only if l divides $2(k-j)$.

Proof. For (1) the idea, in the spirit of GIT quotient equals symplectic quotient [Kirwan 1984; Ness 1984], is to identify the symplectic quotient $\mu^{-1}(0) / T^{2}$ with a Hirzebruch surface as an analytic subspace of $\mathbb{C P}^{1} \times \mathbb{C P}^{2}$.

After shifting by a constant vector $\boldsymbol{a}=\left(a_{1}, a_{2}\right)$ the moment map of the $T^{2}$ action (39) is

$$
\mu(z)=\left(2(k-j)\left|z_{1}\right|^{2}+l\left|z_{3}\right|^{2}+l\left|z_{4}\right|^{2}-a_{1},\left|z_{1}\right|^{2}+\left|z_{2}\right|^{2}-a_{2}\right) .
$$

We need to choose the constant vector $\boldsymbol{a}$ so that 0 is a regular value of $\mu$ for all integers $j$ and $l$ such that $0<j \leq k$ and $0<l \leq k$. Alternatively, it suffices to show that the $T^{2}$ action on $\mu^{-1}(0)$ defined by (39) is locally free. This will be true if we choose $a_{1}, a_{2}>0$ and $a_{1}>2(k-j) a_{2}$. Following [Audin 1994] it is convenient to work with the corresponding $\mathbb{C}^{*} \times \mathbb{C}^{*}$ action on $\mathbb{C}^{2} \backslash\{0\} \times \mathbb{C}^{2} \backslash\{0\}$ given by

$$
z \mapsto\left(\tau^{n_{j}} \zeta z_{1}, \zeta z_{2}, \tau^{m_{j}} z_{3}, \tau^{m_{j}} z_{4}\right),
$$

where $\tau, \zeta \in \mathbb{C}^{*}$. From this we see that the action is free if $z_{1} z_{2} \neq 0$ and locally free with isotropy group $\mathbb{Z}_{m_{j}}$ on the two divisors obtained by setting $z_{1}=0$ and 
$z_{2}=0$, respectively. It is not difficult to see [Lafontaine 1981] that $\left(z_{1}=0\right)=E$ and $\left(z_{2}=0\right)=F$.

We have a commutative diagram

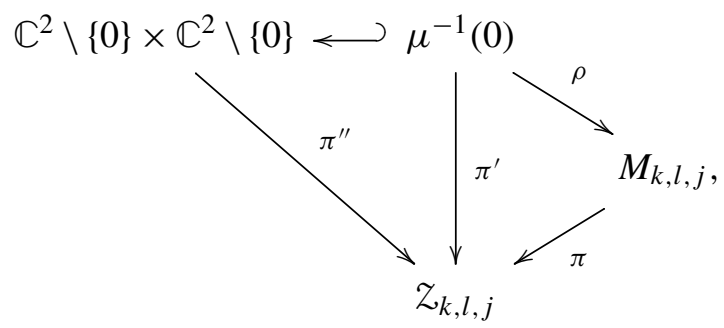

and we want to identify the quotient space $z_{k, l, j}$. We know from the general theory (see [Boyer and Galicki 2008, Chapter 7]) that $z_{k, l, j}$ is a projective algebraic orbifold with an orbifold Kähler structure. Viewing $z_{k, l, j}$ as the $\mathbb{C}^{*} \times \mathbb{C}^{*}$ quotient by the map $\pi^{\prime \prime}$ of diagram (43), we can identify $z_{k, l, j}$ with a subvariety of $\mathbb{C P} \mathbb{P}^{1} \times \mathbb{C P}^{2}$ as in [Hirzebruch 1951; Lafontaine 1981] as follows. If we define homogeneous coordinates in $\mathbb{C P} \mathbb{P}^{1} \times \mathbb{C P}^{2}$ by setting $\left(w_{1}, w_{2}\right)=\left(z_{3}, z_{4}\right)$ and $\left(y_{1}, y_{2}, y_{3}\right)=\left(z_{2}^{m_{j}} z_{3}^{n_{j}}, z_{2}^{m_{j}} z_{4}^{n_{j}}, z_{1}^{m_{j}}\right)$, we see that $z_{k, l, j}$ is represented by the equation

$$
w_{1}^{n_{j}} y_{2}=w_{2}^{n_{j}} y_{1}
$$

As an algebraic variety this identifies $z_{k, l, j}$ with the hypersurface in $\mathbb{C P}^{1} \times \mathbb{C P}^{2}$ defined by (44) which is the original definition of the Hirzebruch surface $S_{n_{j}}$. However, the two divisors in $S_{n_{j}}$ defined by $E=\left(y_{3}=z_{1}^{m_{j}}=0\right)$ and $F=\left(z_{2}=\right.$ $\left.0\left(y_{1}=y_{2}=0\right)\right)$ are both $m_{j}$-fold branch covers with isotropy group $\mathbb{Z}_{m_{j}}$. Thus, we have an orbifold structure on $S_{n_{j}}$ given by (40) which is trivial if and only if $m_{j}=1$ which happens if and only if $l$ divides $2(k-j)$.

Furthermore, it follows from the orbifold Boothby-Wang theorem [Boyer and Galicki 2000b] that $z_{k, l, j}$ has an orbifold Kähler form $\omega_{k, l, j}$ that satisfies $\pi^{*} \omega_{k, l, j}=$ $d \eta_{k, l, j}$. This proves (1). For (2) we note that the orbifold structure is trivial if and only if $m_{j}=1$ which happens if and only if $g_{j}=l$ divides $2(k-j)$.

Notice that on subsets of $\mathcal{J}_{\mathcal{A}}(k, l)$ where $g_{j}$ is independent of $j$, the ramification index $m_{j}$ is also independent of $j$, so the underlying orbifolds are the same. Thus, it is convenient to view $g_{j}$ as a map $g: \mathcal{\partial}_{\mathcal{A}}(k, l) \rightarrow\{1, \ldots, l\}$, and we are interested in the level sets of this map. So we decompose $\mathcal{J}_{\mathcal{A}}(k, l)$ into the level sets of $g$ and then further decompose the level sets according to whether $n_{j}$ is odd or even, that is, we define

$$
\begin{aligned}
g^{-1}(i)_{\mathrm{even}} & =\left\{j \in \mathcal{J}_{\mathcal{A}}(k, l) \mid g_{j}=i, n_{j} \text { is even }\right\}, \\
g^{-1}(i)_{\mathrm{odd}} & =\left\{j \in \mathcal{J}_{\mathcal{A}}(k, l) \mid g_{j}=i, n_{j} \text { is odd }\right\} .
\end{aligned}
$$


We can then decompose the admissible set as a disjoint union

$$
\mathcal{J}_{\mathcal{A}}(k, l)=\bigsqcup_{i=1}^{l} g^{-1}(i)_{\text {even }} \sqcup g^{-1}(i)_{\text {odd }} .
$$

We wish to compute the symplectic form on the base orbifold.

Lemma 4.3. Let $j \in g^{-1}(i)_{\mathrm{even}} \subset \mathcal{J}_{\mathcal{A}}(k, l)$ for a fixed $i \in\{1, \ldots, l\}$ with $g^{-1}(i)_{\mathrm{even}}$ $\neq \varnothing$. Then the symplectic form $\omega_{k, l, i}$ on the quotient $\left(S_{n_{j}}, \Delta_{i}\right)$ is independent of $j$ and satisfies

$$
\left[\omega_{k, l, i}\right]=i \alpha_{E_{0}}+k \alpha_{L} .
$$

Proof. We know that $c_{1}^{\text {orb }}\left(S_{n_{j}}\right)=\left(2 / m_{i}\right) \alpha_{E_{0}}+2 \alpha_{L}$ and this must pull back to $2(k-l) \gamma$. So $\pi^{*} \alpha_{E_{0}}=m_{i} k \gamma$ and $\pi^{*} \alpha_{L}=-l \gamma$. Now the class $\left[\omega_{k, l, i}\right]$ must transcend to 0 on $S^{2} \times S^{3}$. So writing $[\omega]=a \alpha_{E_{0}}+b \alpha_{L}$ and using $i m_{i}=l$, we see that

$$
0=a \pi^{*} \alpha_{E}+b \pi^{*} \alpha_{L}=\left(a m_{i} k-b l\right) \gamma=m_{i}(a k-b i) \gamma .
$$

So taking $a=i$ and $b=k$ gives the result.

Lemma 4.4. Let $j \in g^{-1}(i)_{\text {odd }} \subset \mathcal{J}_{\mathcal{A}}(k, l)$ for a fixed $i \in\{1, \ldots, l\}$ with $g^{-1}(i)_{\text {odd }}$ $\neq \varnothing$. Then $i$ is even and the symplectic form $\omega_{k, l, i}$ on the quotient $\left(S_{n_{j}}, \Delta_{i}\right)$ is independent of $j$ and satisfies

$$
\left[\omega_{k, l, i}\right]=i \alpha_{E_{-1}}+\left(k+\frac{i}{2}\right) \alpha_{L} .
$$

Proof. First $i$ must be even since $i=g_{j}=\operatorname{gcd}(l, 2(k-j))$ and $n_{j}=2(k-j) / i$ is odd. The remainder of the proof is the same as that of Lemma 4.3, except for odd Hirzebruch surfaces we express the symplectic class in term of the exceptional divisor $E_{-1}=E_{0}-\frac{1}{2} L$.

Whenever possible we would like to determine the cardinalities $\# g^{-1}(i)_{\mathrm{even}}$ and $\# g^{-1}(i)_{\text {odd }}$. First, as seen above, $g^{-1}(i)_{\text {odd }}$ is empty when $i$ is odd. Moreover, if $g^{-1}(l)$ is not empty, then $\Delta_{l}=\varnothing$, so the orbifold structure is trivial.

The following lemma is taken from [Karshon 2003].
Lemma 4.5.
(1) $\# g^{-1}(l)_{\mathrm{even}}=\left\lceil\frac{k}{l}\right\rceil$.
(2) $\# g^{-1}(l)_{\text {odd }}=\left\lceil\frac{2 k-l}{2 l}\right\rceil$.

Example 4.6. One obtains the $Y^{p, q}$ of [Gauntlett et al. 2004a] as a special case of Theorem 4.2 by putting $k=l=p$ and defining $q=k-j$. The contact structures are then $\mathcal{D}_{p-q, p+q, p, p}$, and the admissibility conditions boil down to $\operatorname{gcd}(q, p)=1$. Clearly, we have $c_{1}\left(\mathcal{D}_{p-q, p+q, p, p}\right)=0$. When $p$ is odd we have $g_{j}=1, m_{j}=p$, and $S_{n_{j}}=S_{2 q}$, whereas if $p$ is even we have $g_{j}=2, m_{j}=p / 2$ and $S_{n_{j}}=S_{q}$ with $q$ 
odd. So here we have only two nonempty level sets of the map $g$, namely,

$$
\mathcal{J}_{\mathcal{A}}(p, p)= \begin{cases}g^{-1}(1)_{\mathrm{even}}, & \text { if } p \text { is odd, } \\ g^{-1}(2)_{\mathrm{odd}} & \text { if } p \text { is even. }\end{cases}
$$

Since the only admissibility condition is $\operatorname{gcd}(q, p)=1$ and $p>q$, we see that the cardinality $\# \mathcal{J}_{\mathcal{A}}(p, p)=\phi(p)$, where $\phi$ is the well-known Euler phi function. For the orbifold canonical divisor Lemma 4.1 gives

$$
\begin{aligned}
K_{\left(S_{2 q}, \Delta\right)}^{\mathrm{orb}} & =-\frac{2}{p} E-\frac{2(p-q)}{p} L=-\frac{2}{p} E_{0}-2 L, \\
K_{\left(S_{q}, \Delta\right)}^{\mathrm{orb}} & =-\frac{4}{p} E-\frac{2(p-q)}{p} L=-\frac{4}{p} E_{-1}-2 \frac{p+1}{p} L,
\end{aligned}
$$

so these are all log del Pezzo surfaces. The cohomology class of the corresponding symplectic forms is

$$
\left[\omega_{p, p, 1}\right]=\alpha_{E_{0}}+p \alpha_{L}
$$

on the even orbifold Hirzebruch surface $\left(S_{2 q}, \Delta\right)$ with ramification index $m_{1}=p$ when $p$ is odd. For even $p$ we have

$$
\left[\omega_{p, p, 2}\right]=2 \alpha_{E_{-1}}+(p+1) \alpha_{L}
$$

on the odd orbifold Hirzebruch surface $\left(S_{q}, \Delta\right)$ with ramification index $m_{2}=p / 2$. Note that in both cases there are precisely $\phi(p)$ values taken on by $q$. Note also that $p=2$ implies $q=1$ only, and that $m_{2}=1$, so we have a trivial orbifold structure on $\left(S_{1}, \varnothing\right)=\widetilde{\mathbb{C P}^{2}}$. A relation between the $Y^{p, q}$ toric contact structures and Hirzebruch surfaces was noted by Abreu [2010].

Except for the $Y^{p, q}$ case of Example 4.6, we do not have a general formula for the cardinalities $\# g^{-1}(i)$ for $i \neq l$. Specific cases, of course, are easy to work out.

Example 4.7. Consider the case $(k, l)=(9,8)$. We compute $\mathcal{J}_{\mathcal{A}}(9,8)$. The possible values of $j$ are all odd with $j \leq 9$, and these all satisfy $\operatorname{gcd}(8,18-j)=1$. Next, we determine $g_{j}=\operatorname{gcd}(8,2(9-j))$ and $n_{j}=2(9-j) / g$. So we have $g^{-1}(8)_{\text {even }}=$ $\{j=1,9\}$ with a trivial orbifold $\left(m_{8}=1\right)$ on the Hirzebruch surfaces $S_{2}$ and $S_{0}$, respectively. We also have $g^{-1}(8)_{\text {odd }}=\{j=5\}$ with a trivial orbifold on $S_{1}$, and $g^{-1}(4)_{\text {odd }}=\{j=3,7\}$ with $m_{4}=2$ on the odd Hirzebruch surfaces $S_{3}$ and $S_{1}$, respectively. Notice that the cardinalities of $g^{-1}(8)_{\text {even }}$ and $g^{-1}(8)_{\text {odd }}$ agree with

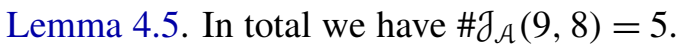

Toric contact structures on $\boldsymbol{X}_{\infty}$. For $X_{\infty}$ we consider $\boldsymbol{p}=(j, 2 k-j+1, l, l)$ with $0<j \leq k$. Here we have $c_{1}\left(\mathcal{D}_{j, 2 k-j+1, l, l}\right)=(2(k-l)+1) \gamma$. We consider the Reeb vector field $R_{j, k, l}^{\infty}=(2 k-j+1) H_{1}+j H_{2}+l H_{3}+l H_{4}$, which is clearly positive. The 
$T^{2}$ action generated by this vector field and $L_{p}=j H_{1}+(2 k-j+1) H_{2}-l H_{3}-l H_{4}$ is

$$
z \mapsto\left(e^{i((2 k-j+1) \phi+j \theta)} z_{1}, e^{i(j \phi+(2 k-j+1) \theta)} z_{2}, e^{i l(\phi-\theta)} z_{3}, e^{i l(\phi-\theta)} z_{4}\right) .
$$

Making the substitutions $\psi=\phi-\theta$ and $\chi=j \psi+(2 k+1) \theta$ gives the action

$$
z \mapsto\left(e^{(i(2 k-2 j+1) \psi+\chi)} z_{1}, e^{i \chi} z_{2}, e^{i l \psi} z_{3}, e^{i l \psi} z_{4}\right) .
$$

Similarly to the previous section, we define $g_{j}=\operatorname{gcd}(l, 2 k-2 j+1)$ and write $2 k-2 j+1=n_{j} g_{j}$ and $l=m_{j} g_{j}$. Then $\operatorname{gcd}\left(m_{j}, n_{j}\right)=1$.

Theorem 4.8. Consider the contact manifold $\left(X_{\infty}, \mathcal{D}_{j, 2 k-j+1, l, l}\right)$ where $1 \leq j \leq k$ satisfies $\operatorname{gcd}(j, l)=\operatorname{gcd}(2 k-j+1, l)=1$. Then we have

(1) The quotient space by the circle action generated by the Reeb vector field $R=(2 k-j+1) H_{1}+j H_{2}+l H_{3}+l H_{4}$ is the Kähler orbifold $\left(S_{n_{j}}, \Delta ; \omega_{k, l, j}\right)$ where $S_{n_{j}}$ is an odd Hirzebruch surface, $\Delta$ is the branch divisor, with

$$
\Delta=\left(1-\frac{1}{m_{j}}\right)\left(z_{1}=0\right)+\left(1-\frac{1}{m_{j}}\right)\left(z_{2}=0\right),
$$

and $\omega_{k, l, j}$ is an orbifold symplectic form satisfying $\pi^{*} \omega_{k, l, j}=d \eta_{k, l, j}$, where $\eta_{k, l, j}$ is the contact 1-form representing $\mathcal{D}_{j, 2 k-j+1, l, l}$ whose Reeb vector field is $R$. Here the integers $l, g_{j}, n_{j}$, and $m_{j}$ are all odd.

(2) The orbifold structure is trivial $(\Delta=\varnothing)$ if and only if l divides $2 k-j+1$.

Proof. The proof is essentially the same as that of Theorem 4.2. The details are left to the reader.

We denote the set of $j=1, \ldots, k$ such that $\boldsymbol{p}=(j, 2 k-j+1, l, l)$ is admissible by $\mathcal{J}_{\mathcal{A}}^{\infty}=\mathcal{J}_{\mathcal{A}}^{\infty}(k, l)$. Since the integers $g_{j}$ and $n_{j}$ are both odd for all $j \in \mathcal{J}_{\mathcal{A}}(k, l)$, the map $g$ maps the set $\mathcal{J}_{\mathcal{A}}(k, l)$ to the set of positive odd integers less than or equal to $l$. Thus, we have

$$
\mathcal{\partial}_{\mathcal{A}}^{\infty}(k, l)=\bigsqcup_{\text {odd } i=1}^{l} g^{-1}(i) .
$$

Similarly to Lemmas $4.3,4.4$, and 4.5 we find the following.

Lemma 4.9. Let $j \in g^{-1}(i) \subset \mathcal{J}_{\mathcal{A}}^{\infty}(k, l)$. Then the symplectic form $\omega_{k, l, i}$ on the quotient $S_{n_{j}}$ is independent of $j$ and satisfies

$$
\left[\omega_{k, l, i}\right]=i \alpha_{E_{-1}}+\left(k+\frac{i+1}{2}\right) \alpha_{L} .
$$

Furthermore, $\# g^{-1}(l)=\lceil(2 k-l+1) / 2 l\rceil$. 
Example 4.10. We consider the analogue on $X_{\infty}$ of Example 4.6, so $(k, l)=$ $(p, p)$ with $p$ odd and $j=p-q$. The contact structure is $\mathcal{D}_{p-q, p+q+1, p, p}$ with $c_{1}\left(\mathcal{D}_{p-q, p+q+1, p, p}\right)=1$. The admissibility conditions are $\operatorname{gcd}(q, p)=1=\operatorname{gcd}(q+$ $1, p)$. The function $g$ satisfies $g_{j}=\operatorname{gcd}(2 q+1, p)$. If $p$ is prime then the set of admissible $q$ is $\{1, \ldots, p-2\}$, and $g=1$ except when $q=(p-1) / 2$ in which case $g=p$. The latter is smooth and corresponds to the trivial orbifold $\left(S_{0}, \varnothing\right)$. This has symplectic class

$$
\left[\omega_{p, p}\right]=p \alpha_{E_{-1}}+\frac{3 p+1}{2} \alpha_{L},
$$

whereas the $p-3$ elements in $g^{-1}(1)$ have symplectic class

$$
\left[\omega_{p, p}\right]=\alpha_{E_{-1}}+(p+1) \alpha_{L} .
$$

For a case when $p$ is not prime consider $p=9$. Then $\mathcal{J}_{\mathcal{A}}^{\infty}(9,9)=\{2,5,8\}$ which corresponds to $q=7,4,1$. We see that $g^{-1}(9)=\{5\}$ giving the smooth first Hirzebruch surface $\left(S_{1}, \varnothing\right)$ with symplectic class $\left[\omega_{9,9,9}\right]=9 \alpha_{E_{-1}}+14 \alpha_{L}$, while $g^{-1}(3)=\{2,8\}$ giving the orbifold Hirzebruch surfaces $\left(S_{5}, \Delta\right)$ with $m_{2}=3$, and $\left(S_{1}, \Delta\right)$ with $m_{8}=3$, respectively, with symplectic class $\left[\omega_{9,9,3}\right]=3 \alpha_{E_{-1}}+11 \alpha_{L}$.

Equivalent contact structures. Here we show that certain inequivalent toric contact structures are equivalent as contact structures. The proof uses the fact that the identity map $\left(S_{n}, \Delta_{m}\right) \rightarrow\left(S_{n}, \varnothing\right)$ is a Galois cover, and combines this with the work of Karshon [1999; 2003] for the smooth case.

Theorem 4.11. Consider the toric contact structures $\left(S^{2} \times S^{3}, \mathcal{D}_{j, 2 k-j, l, l}\right)$ and $\left(X_{\infty}, \mathcal{D}_{j, 2 k-j+1, l, l}\right)$ of Theorems 4.2 and 4.8 , respectively.

(1) For each fixed $1 \leq i \leq l$, the contact structures $\mathcal{D}_{j, 2 k-j, l, l}$ are $T^{2}$-equivariantly isomorphic for all $j \in g^{-1}(i)_{\mathrm{even}}$, and the contactomorphism group

$$
\mathfrak{C o n}\left(\mathcal{D}_{j, 2 k-j, l, l}\right)
$$

has at least $\mathrm{Hg}^{-1}(i)_{\mathrm{even}}$ conjugacy classes of maximal tori of dimension three.

(2) For each fixed $1 \leq i \leq l$, the contact structures $\mathcal{D}_{j, 2 k-j, l, l}$ are $T^{2}$-equivariantly isomorphic for all $j \in g^{-1}(i)_{\text {odd }}$, and the contactomorphism group

$$
\mathfrak{C o n}\left(\mathcal{D}_{j, 2 k-j, l, l}\right)
$$

has at least $\# g^{-1}(i)_{\mathrm{odd}}$ conjugacy classes of maximal tori of dimension three.

(3) For each fixed $1 \leq i \leq l$, the contact structures $\mathcal{D}_{j, 2 k-j+1, l, l}$ are $T^{2}$-equivariantly isomorphic for all $j \in g^{-1}(i) \subset \mathcal{J}_{\mathcal{A}}^{\infty}(k, l)$, and the contactomorphism group $\mathfrak{C o n}\left(\mathcal{D}_{j, 2 k-j+1, l, l}\right)$ has at least $\# g^{-1}(i)$ conjugacy classes of maximal tori of dimension three. 
(4) The $T^{2}$-equivariantly isomorphic contact structures given in (1)-(3) are not $T^{3}$-equivariantly isomorphic.

Proof. The proofs of (1)-(3) are quite analogous, so we give the details for (1) only. By Theorem 4.2 the contact structure is the orbifold Boothby-Wang construction over the symplectic orbifold $\left(S_{n_{j}}, \omega_{k, l, j}\right)$ and by Lemma 4.3 the form $\omega_{k, l, j}$ only depends on $g_{j}=i$. Then for each $j \in g^{-1}(i)_{\text {even }}$ we consider the Galois cover $\mathbb{1}_{n_{j}}:\left(S_{n_{j}}, \Delta_{m_{i}}\right) \rightarrow\left(S_{n_{j}}, \varnothing\right)$ with $n_{j}$ even, and both spaces having the same Kähler form, namely $\omega_{k, l, i}$ of Lemma 4.3. Now Karshon [2003] shows that $\left(S_{n_{j}}, \varnothing\right)$ and $\left(S_{n^{\prime}}, \varnothing\right)$ are $S^{1}$-equivariantly symplectomorphic with the same symplectic form $\omega_{k, l, i}$ (but not the same Kähler structure) as long as $0 \leq j^{\prime}=j-2 r$ for some nonnegative integer $r$. We denote such a symplectomorphism by $K$. Now we have a commutative diagram,

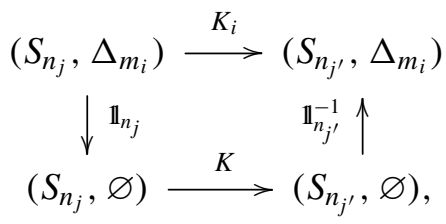

which defines the upper horizontal arrow $K_{i}$ and shows that it too is an $S^{1}$-equivariant symplectomorphism. We claim that $K_{i}$ is also an orbifold diffeomorphism. This follows from Lemma 4.12. But then, as shown in [Lerman 2003b; Boyer 2013], this symplectomorphism lifts to a $T^{2}$-equivariant contactomorphism.

Here, and hereafter, by $g^{-1}(i)$ we mean any of the three sets $g^{-1}(i)_{\mathrm{even}}, g^{-1}(i)_{\text {odd }}$, or $g^{-1}(i) \subset \mathcal{J}_{\mathcal{A}}^{\infty}(k, l)$. Since our contact structures are independent of $j \in g^{-1}(i)$ up to isomorphism, we now denote the contact structures of items (1), (2), and (3) by $\mathcal{D}_{k, l, i, e}, \mathcal{D}_{k, l, i, o}$, and $\mathcal{D}_{k, l, i, \infty}$, respectively. To prove (4) we first notice that as in [Karshon 2003] the orbifold symplectomorphism $K_{i}$ is only $S^{1}$-equivariant, not $T^{2}$-equivariant. So the corresponding 2-tori belong to different conjugacy classes in the group $\mathfrak{H a m}\left(\left(\mathcal{B}, \Delta_{m_{i}}\right), \omega_{k, l, i}\right)$ of Hamiltonian symplectomorphisms, where $\mathcal{B}$ is the symplectic orbifold $\left(\left(S^{2} \times S^{2}, \Delta_{m_{i}}\right) \omega_{k, l, i}\right)$ or $\left(\left(X_{\infty}, \Delta_{m_{i}}\right) \omega_{k, l, i}\right)$ as the case may be. But then by [Boyer 2013, Theorem 6.4] these lift to nonconjugate 3-tori in $\mathfrak{C o n}\left(\mathcal{D}_{k, l, i, e}\right)$. Hence, the contact structures $\mathcal{D}_{j, 2 k-j, l, l}$ for different $j \in g^{-1}(i)$ are inequivalent as toric contact structures. The same holds for $\mathcal{D}_{j, 2 k-j+1, l, l}$.

Lemma 4.12. The Karshon symplectomorphism $K$ of diagram (52) leaves the divisors $\left(z_{1}=0\right)$ and $\left(z_{2}=0\right)$ invariant.

Proof. The $T^{2}$ action on any orbifold Hirzebruch surface $\left(S_{n}, \Delta_{m}\right)$ can be taken as

$$
\left(\left[w_{1}, w_{2}\right] \times\left[y_{1}, y_{2}, y_{3}\right]\right) \mapsto\left(\left[\tau w_{1}, w_{2}\right] \times\left[\tau^{n} y_{1}, y_{2}, \rho y_{3}\right]\right),
$$

where, as in the proof of Theorem 4.2, the coordinates are $\left(w_{1}, w_{2}\right)=\left(z_{3}, z_{4}\right)$ and $\left(y_{1}, y_{2}, y_{3}\right)=\left(z_{2}^{m} z_{3}^{n}, z_{2}^{m} z_{4}^{n}, z_{1}^{m}\right)$. By Proposition 4.1 of [Karshon 1999] $K$ is 
an $S^{1}$-equivariant symplectomorphism, where the $S^{1}$ is that generated by $\rho$, and the fixed point set of this action is the disjoint union $\left(z_{1}=0\right) \sqcup\left(z_{2}=0\right)$. By Proposition 4.3 of the same work, $K$ also intertwines the two $S^{1}$ moment maps. But in both cases these are represented by $\mu_{S}(z)=\left|z_{1}\right|^{2}$. So the divisors $\left(z_{1}=0\right)$ and $\left(z_{2}=0\right)$ are left invariant separately by $K$.

Remark 4.13. Using the Delzant theorem for symplectic orbifolds in [Lerman and Tolman 1997], it is straightforward to construct the labeled polytope corresponding to the symplectic orbifold $\left(\left(S_{n}, \Delta_{m}\right), \omega_{k, l, i}\right)$. It is the labeled Hirzebruch trapezoid shown here (with label $m$ on the two vertical axes):

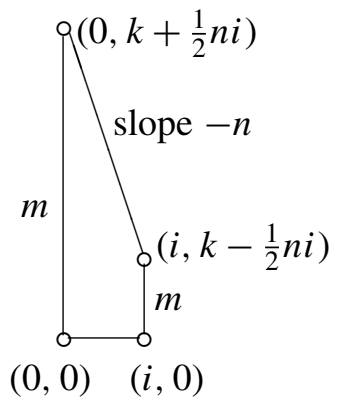

The Galois cover $\mathbb{1}_{S_{n}}:\left(S_{n}, \Delta_{m}\right) \rightarrow\left(S_{n}, \varnothing\right)$ induces a map on this Hirzebruch trapezoid that simply removes the labels on the vertical edges. This implies that the corresponding Karshon graphs [1999] are the same. Hence, Theorem 4.1 of [Karshon 1999] easily generalizes to the types of orbifolds considered here, and the symplectomorphism $K_{i}$ in diagram (52) can be constructed directly from this.

Inequivalence of contact structures. As discussed previously the inequivalence of contact structures is detected first by the first Chern class $c_{1}(\mathcal{D})$ and then by contact homology. The contact structures $\mathcal{D}_{j, 2 k-j, l, l}$ and $\mathcal{D}_{j, 2 k-j+1, l, l}$ are clearly inequivalent since they live on different manifolds, so adopting Proposition 3.11 to our current notation we have

Theorem 4.14. The contact structures $\mathcal{D}_{j, 2 k-j, l, l}$ and $\mathcal{D}_{j^{\prime}, 2 k^{\prime}-j^{\prime}, l^{\prime}, l^{\prime}}$ and $\mathcal{D}_{j, 2 k-j+1, l, l}$ and $\mathcal{D}_{j^{\prime}, 2 k^{\prime}-j^{\prime}+1, l^{\prime}, l^{\prime}}$ are inequivalent if $k^{\prime} \neq k$.

Remark 4.15. Unfortunately, combining Theorems 4.11 and 4.14 does not answer our equivalence problem completely even in our restrictive cases. For example, it would be nice to know that the $\mathcal{D}_{j, 2 k-j, l, l}$ are all contact equivalent as $j$ runs through all admissible values from 1 to $k$. However, our equivalence statement in Theorem 4.11 only assures equivalence on the level sets of the map $g$, that is, if we fix $i \in\{1, \ldots, l\}$, then $\mathcal{D}_{j, 2 k-j, l, l}$ are equivalent for all $j \in g^{-1}(i)$. Nevertheless, this is enough to give a complete answer to the equivalence problem for the $Y^{p, q}$ of [Gauntlett et al. 2004a] which in our notation is $\mathcal{D}_{p-q, p+q, p, p}$. See Corollary 5.5. 
The general case with vanishing first Chern class $c_{1}(\mathcal{D})$ was studied in [Cvetič et al. 2005; Martelli and Sparks 2005] where it was shown that all the toric contact structures admit a compatible Sasaki-Einstein metric. These depend on three parameters $a, b$, and $c$ with values in $\mathbb{Z}^{+}$which in our notation is given by $\mathcal{D}_{a, b, c, a+b-c}$. Except for the subclass $Y^{p, q}$ these fall outside of the scope of our analysis.

Another statement of contact inequivalence is obtained as an immediate consequence of Theorem 3.13, namely:

Corollary 4.16. The contact structures on $S^{2} \times S^{3}$ described by items (1) and (2) of Theorem 4.11 are inequivalent.

\section{Applications to Sasakian geometry}

In this final section we give some pertinent applications of our results to Sasakian geometry. Recall the contact Delzant-type result [Boyer and Galicki 2000a] that every toric contact structure of Reeb type admits a compatible Sasakian structure.

Sasaki cones and the Sasaki bouquet. Since we are dealing with toric geometry, the Sasaki cones in this paper all have dimension three. So it follows from [Boyer and Galicki 2008, Theorem 8.1.14] that all our Sasakian structures must be either positive or indefinite. Our first result says that all our Sasaki cones have a positive Sasakian structure.

Corollary 5.1. Every toric contact structure on an $S^{3}$-bundle over $S^{2}$ can be realized as an orbifold fibration over $\mathbb{C P}\left(\bar{p}_{1}, \bar{p}_{2}\right) \times \mathbb{C P}\left(\bar{p}_{3}, \bar{p}_{4}\right)$ for some quadruple of positive integers $\left(p_{1}, p_{2}, p_{3}, p_{4}\right)$ satisfying $\operatorname{gcd}\left(p_{i}, p_{j}\right)=1$ for $i=1,2$ and $j=3,4$ and $\left(p_{1}, p_{2}, p_{3}, p_{4}\right)=\left(k \bar{p}_{1}, k \bar{p}_{2}, l \bar{p}_{3}, l \bar{p}_{4}\right)$. Thus, every toric contact structure on an $S^{3}$-bundle over $S^{2}$ admits a ray of positive Sasakian structures in its Sasaki cone. Moreover, the subspace of positive Sasakian structures is open in the Sasaki cone.

Proof. The first statement follows from Lemmas 2.2 and 2.5, while the second statement follows from the fact that the base orbifold $\mathbb{C P}\left(\bar{p}_{1}, \bar{p}_{2}\right) \times \mathbb{C P}\left(\bar{p}_{3}, \bar{p}_{4}\right)$ is log del Pezzo. The last statement follows from the fact that having a positive representative of the basic first Chern class $c_{1}\left(\mathcal{F}_{R}\right)$ is an open condition.

Proposition 5.2. Consider the toric contact structure $\mathcal{D}_{j, 2 k-j, l, l}$ on $S^{2} \times S^{3}$ and the toric contact structure $\mathcal{D}_{j, 2 k-j+1, l, l}$ on $X_{\infty}$. Choose the Reeb vector field $R=$ $(2 k-j) H_{1}+j H_{2}+l H_{3}+l H_{4}$ on $\left(S^{2} \times S^{3}, \mathcal{D}_{j, 2 k-j+1, l, l}\right)$ and $R=(2 k-j+1) H_{1}+$ $j \mathrm{H}_{2}+\mathrm{lH}_{3}+l \mathrm{H}_{4}$ on $\left(\mathrm{X}_{\infty}, \mathcal{D}_{j, 2 k-j+1, l, l}\right)$. Then the corresponding Sasakian structure is positive if and only if $l>k-j$ in the former case, and $2 l>2(k-j)+1$ in the latter case. In particular, if $l \leq k-j($ or $2 l>2(k-j)+1)$, then the toric contact structure $\mathcal{D}_{j, 2 k-j, l, l}$ (or $\mathcal{D}_{j, 2 k-j+1, l, l}$ ) has both a positive and indefinite Sasakian structure in its Sasaki cone. 
Proof. First we note as mentioned above that any Sasakian structure in a Sasaki cone of dimension greater than one is either positive or indefinite. By Corollary 5.1 any toric contact structure on an $S^{3}$-bundle over $S^{2}$ has a positive Sasakian structure, and we see that Lemma 4.1 states that $\left(S_{n_{j}}, \Delta_{m_{j}}\right)$ is log del Pezzo if and only if $2 m>n$. For $\mathcal{D}_{j, 2 k-j, l, l}$ this becomes $2 l=2 m_{j} g_{j}>n_{j} g_{j}=2(k-j)$, whereas for $\mathcal{D}_{j, 2 k-j+1, l, l}$ it is $2 l=2 m_{j} g_{j}>n g_{j}=2 k-2 j+1$. The last result then follows from the fact that a quasiregular Sasakian structure is positive if and only if its orbifold quotient is log Fano [Boyer and Galicki 2008, Chapter 7].

Concerning Sasaki bouquets we note that actually more is true than is proven in Theorem 4.11. Since the base orbifolds $\left(S_{n_{j}}, \Delta_{m_{i}}\right)$ have the same symplectic form $\omega_{k, l, i}$ for all $j \in g^{-1}(i)$, this lifts to a contactomorphism $\varphi: \mathcal{D}_{j, 2 k-j, l, l} \rightarrow$ $\mathcal{D}_{j^{\prime}, 2 k-j^{\prime}, l, l}$ such that $\varphi^{*} \eta_{j^{\prime}, 2 k-j^{\prime}, l, l}=\eta_{j, 2 k-j, l, l}$. Since the reduction process carries a preferred complex structure $J$ along with it, the different indices $j$ represent different transverse complex structures $J$. So by using the contactomorphisms $\varphi$ for each admissible $j$, we have the following.

Corollary 5.3. The contact structures $\mathcal{D}_{k, l, i, e}$ and $\mathcal{D}_{k, l, i, o}$ on $S^{2} \times S^{3}$ and $\mathcal{D}_{k, l, i, \infty}$ on $X_{\infty}$ each admit a Sasaki bouquet $\mathfrak{B}_{N}$ of toric Sasakian structures with $N=$ $\# g^{-1}(i)$. Furthermore, the intersection $\bigcap_{j} \kappa\left(\mathcal{D}, J_{j}\right)$ of all the Sasaki cones is an open subset of the Lie algebra $\mathfrak{t}_{2}$ of a two-dimensional torus.

Example 5.4. Consider the contact structure $\mathcal{D}_{12,8,2, o}$ on $S^{2} \times S^{3}$. The admissible $j$ 's are $j=1,3,5,7$ and $g_{j}=2$ for all $j$. So Theorem 4.11(ii) gives a $T^{2}$-equivariant contact equivalence of the $T^{3}$-equivariantly inequivalent toric contact structures

$$
\mathcal{D}_{1,23,8,8} \approx \mathcal{D}_{3,21,8,8} \approx \mathcal{D}_{5,19,8,8} \approx \mathcal{D}_{7,17,8,8}
$$

This implies that the number of conjugacy classes $\mathfrak{n}\left(\mathcal{D}_{12,8,2, o}, 3\right)$ of 3-tori in $\operatorname{Con}\left(\mathcal{D}_{12,8,2, o}\right)$ is at least 4. Furthermore, by Theorem 4.2(3) the induced Sasakian metrics are positive for $\mathcal{D}_{5,19,8,8}$ and $\mathcal{D}_{7,17,8,8}$, whereas they are indefinite for the remaining two.

Another contact structure with the same first Chern class as $\mathcal{D}_{12,8,2, o}$, namely $c_{1}=8 \gamma$, is $\mathcal{D}_{14,10,2, o}$. This consists of the two $T^{3}$-equivariantly inequivalent toric contact structures $\mathcal{D}_{1,27,10,10}$ and $\mathcal{D}_{7,21,10,10}$, but only for the latter is the induced Sasakian structure positive. In this case we have $\mathfrak{n}\left(\mathcal{D}_{14,10,2, o}, 3\right) \geq 2$. Moreover, it follows from Theorem 4.14 that the contact structures $\mathcal{D}_{12,8,2, o}$ and $\mathcal{D}_{14,10,2, o}$ are inequivalent.

As mentioned previously there is one subclass of contact structures on $S^{2} \times S^{3}$ where a complete solution to the equivalence problem can be obtained, and they are all known to admit extremal (actually Sasaki-Einstein) metrics. 
Corollary 5.5. The contact structures $Y^{p, q}$ and $Y^{p^{\prime}, q^{\prime}}$ on $S^{2} \times S^{3}$ are inequivalent if and only if $p^{\prime} \neq p$. Furthermore, the isotopy class of the contact structures defined by $Y^{p, 1}$ admits a $\phi(p)$-bouquet $\mathfrak{B}_{\phi(p)}\left(Y^{p, q}\right)$ such that each of the $\phi(p)$ Sasaki cones admits a unique Sasaki-Einstein metric. Moreover, these Einstein metrics are nonisometric as Riemannian metrics.

Proof. Applying Theorem 4.11 to Example 4.6 shows that $Y^{p, q}$ is contactomorphic to $Y^{p, 1}$ for all admissible $q$. But Abreu and Macarini [2012] show that the underlying contact structures of $Y^{p, 1}$ and $Y^{p^{\prime}, 1}$ are inequivalent if $p^{\prime} \neq p$. (This also follows from Proposition 3.11.) By Corollary 5.3 there are precisely $\phi(p)$ Sasaki cones in the bouquet. The fact that there is a Sasaki-Einstein metric in the Sasaki cone for each $Y^{p, q}$ was first shown in [Gauntlett et al. 2004a] while its uniqueness in the Sasaki cone is proved in [Cho et al. 2008].

To prove the last statement, suppose to the contrary that the Sasaki-Einstein metrics $g_{q}$ and $g_{q^{\prime}}$ are isometric, that is, there is a diffeomorphism $\psi$ of $S^{2} \times S^{3}$ such that $\psi^{*} g_{q^{\prime}}=g_{q}$. Then by a theorem of Tanno [1970] (see also [Boyer and Galicki 2008, Lemma 8.1.17]) the transformed Sasakian structure $\mathcal{S}^{\psi}$ is either $\mathcal{S}_{q}$ itself or its conjugate Sasakian structure $\mathcal{S}_{q}^{c}=\left(-R_{q},-\eta_{q},-\Phi_{q}, g_{q}\right)$. In either case $\psi$ is a contactomorphism from $Y^{p, q}$ to $Y^{p, q^{\prime}}$ satisfying

$$
\psi^{-1} \circ \Phi_{q^{\prime}} \circ \psi= \pm \Phi_{q} .
$$

But this implies that the corresponding 3-tori are conjugate, which contradicts Theorem 4.11(4).

Example 5.6. The analogues of the $Y^{p, q}$ 's on the nontrivial bundle $X_{\infty}$ are described in Example 4.10. For simplicity we consider only the case when $p$ is an odd prime, in which case there are $p-3$ admissible values for $q$, namely $1, \ldots,(p-1) / 2-1,(p-1) / 2+1, \ldots, p-2$. These inequivalent toric structures are $T^{2}$-equivariantly equivalent contact structures by Theorem 4.11(3) and their induced Sasakian structures are all positive by Theorem 4.8(3). Moreover, the contactomorphism group of this contact structure has at least $p-3$ maximal tori of dimension three.

Some remarks concerning extremal Sasakian structures. As with Kähler geometry it is of interest to determine the most preferred Sasakian metrics, and as in Kähler geometry it seems reasonable to study the critical points of the (now transverse) Calabi functional [Boyer et al. 2008; 2009]. In [Boyer 2011b] the first author described bouquets of extremal Sasakian structures on $S^{3}$-bundles over $S^{2}$, and the existence of extremal Sasakian metrics on $X_{\infty}$ was proven. It is not our intention here to delve much further into the existence of such extremal Sasakian structures, but rather to discuss briefly their relation to our current work.

Corollary 5.3 gives a partial generalization of Theorems 4.1 and 4.2 of [Boyer 
2011b]. In this reference it was shown that when the quotient by the Reeb vector field is a smooth manifold, each Sasaki cone in a bouquet admits an extremal Sasakian metric. This follows from well-known work of Calabi. It would be interesting to generalize this to the orbifold case by generalizing the method of [Ghigi and Kollár 2007] to extremal metrics.

As with toric symplectic structures, all toric contact structures of Reeb type admit a compatible Sasakian metric [Boyer and Galicki 2000a]. Furthermore, in our present situation we have:

Corollary 5.7. Every toric contact structure on an $S^{3}$ bundle over $S^{2}$ admits extremal Sasakian metrics with positive Ricci curvature.

Proof. By Corollary 5.1 every toric contact structure on an $S^{3}$ bundle over $S^{2}$ can be realized as an orbifold fibration over a product of weighted projective spaces $\mathbb{C P}\left(\bar{p}_{1}, \bar{p}_{2}\right) \times \mathbb{C P}\left(\bar{p}_{3}, \bar{p}_{4}\right)$ and have positive Sasakian structures. By a result of Bryant [2001] all weighted projective spaces admit Bochner-flat metrics and these are extremal [David and Gauduchon 2006], and the product of extremal Kähler metrics is extremal. So these extremal Kähler orbifold metrics lift to extremal Sasakian metrics [Boyer et al. 2008] which, since $\mathbb{C P}\left(\bar{p}_{1}, \bar{p}_{2}\right) \times \mathbb{C P}\left(\bar{p}_{3}, \bar{p}_{4}\right)$ is $\log$ del Pezzo, will have a deformation to a Sasakian metric with positive Ricci curvature by [Boyer and Galicki 2008, Theorem 7.5.31]. Moreover, it follows from a theorem of Calabi [1985] that the toric symmetry is retained by these metrics.

Corollary 5.7 implies that each Sasaki cone in every Sasaki bouquet $\mathfrak{B}_{N}$ of toric contact structures on an $S^{3}$ bundle over $S^{2}$ admits extremal Sasakian metrics of positive Ricci curvature. Since the moment cone of any $S^{3}$ bundle over $S^{2}$ has exactly four facets, recent results of Legendre [2011a; 2011b] show that every toric contact structure on an $S^{3}$ bundle over $S^{2}$ admits at least one and at most seven distinct rays in the Sasaki cone consisting of Sasakian structures whose metrics have constant scalar curvature. Moreover, she shows that for the Wang-Ziller manifolds $M_{k, l}^{1,1}$ with $k>5 l$ there exist two distinct rays in the Sasaki cone whose Sasakian metrics have constant scalar curvature. This corresponds to the case $\left(\bar{p}_{1}, \bar{p}_{2}\right)=1=\left(\bar{p}_{3}, \bar{p}_{4}\right)$ of Lemma 2.5 .

An interesting question which appears to be unanswered at this time is whether any Sasaki cones on these toric contact structures are exhausted by extremal Sasaki metrics. There are only a few known cases where this occurs, namely, the standard CR structure on the spheres $S^{2 n+1}$ [Boyer et al. 2008], the Heisenberg group [Boyer 2009], and $T^{2}$-invariant contact structures of Reeb type on $S^{3}$-bundles over Riemann surfaces [Boyer and Tønnesen-Friedman 2014; 2013] with genus $1 \leq g \leq 4$. However, when $g=0$ we suspect that by using the admissible construction method of [Boyer and Tønnesen-Friedman 2014] the subclass of Sasaki structures considered here will each have an extremal representative. 


\section{Appendix: Orbifold Gromov-Witten invariants}

In this appendix, for the convenience of the reader, we lay out some framework and definitions for Gromov-Witten invariants and the so-called Gromov-Witten potential for compact symplectic manifolds and orbifolds. In this paper we only consider the genus-0 invariants. The Gromov-Witten invariants that we are interested in occur in the base orbifold $z$ of an orbibundle $\pi: M \rightarrow z$ with $\operatorname{dim}(M)=5$. Hence we are in the semipositive case and we can define the Gromov-Witten invariants as in [McDuff and Salamon 2004]. Our version of Gromov-Witten theory for symplectic orbifolds comes from [Chen and Ruan 2002]. The main difference here is that our marked points, and hence our cohomology classes taken as arguments for the invariant, have constraints determining in which orbifold stratum the curves in question lie. This is an issue since generally some homology classes may live in several strata.

Roughly speaking a Gromov-Witten invariant is a count of rigid $J$-holomorphic curves representing a homology class $A \in H_{2}(M, \mathbb{Z}) /($ torsion) in general position with marked points in a symplectic manifold $M$ for which the marked points are mapped into the Poincaré duals of certain cohomology classes. For example we may ask how many spheres (or lines) intersect two generic points in $\mathbb{C} \mathbb{P}^{n}$. In this case we have two marked points, a top cohomology class, and for $A$ the class of a line, $[L]$.

To make this precise let $(M, \omega)$ be a compact symplectic manifold, and let $J$ be an $\omega$-compatible almost-complex structure. Consider the moduli space

$$
\mathcal{M}_{0, k}^{A}(M, J)
$$

of genus- 0 stable $J$-holomorphic curves into $M$ representing the class $A$ and assume here that we have regularity of the relevant linearized Cauchy-Riemann operator for the class $A$, either via some circumstances or by some sort of abstract perturbation argument. Note also that when we discuss Gromov-Witten theory for compact symplectic manifolds we will consider only somewhere injective curves. We define maps

$$
e v_{j}: \mathcal{M}_{0, k}^{A}(M, J) \rightarrow M \quad \text { and } \quad e v: \mathcal{M}_{0, k}^{A}(M, J) \rightarrow M^{\times k}
$$

by evaluation at the marked points.

By semipositivity the evaluation map represents a submanifold of $M^{\times k}$ of dimension

$$
2 n+\left\langle 2 c_{1}(M), A\right\rangle+2 k+6 .
$$

Now we define the Gromov-Witten invariant as a homomorphism

$$
\mathrm{GW}_{A, k}^{M}: H^{*}(M)^{\otimes k} \otimes H_{*}\left(\mathcal{M}_{0, k}^{A}(M . J)\right) \rightarrow \mathbb{Q}
$$


encoded formally as the integral

$$
\mathrm{GW}_{A, k}^{M}\left(\alpha_{1}, \ldots, \alpha_{k}\right):=\int_{\mathcal{M}_{0, k}^{A}(M . J)} e v_{1}^{*} \alpha_{1} \cup \ldots \cup e v_{k}^{*} \alpha_{k} \cup \pi^{*}\left[\mathcal{M}_{0, k}^{A}(M . J)\right] .
$$

This is the definition for manifolds. This definition can be used without the semipositivity condition as long as there is a construction of an appropriate object on which to integrate. Since we will be working in dimension four this will not be an issue.

To extend this definition to orbifolds, there are issues with the definitions of $J$-holomorphic curves, since the idea of a map between orbifolds can be a rather sticky issue. We content ourselves here with knowing that we have a notion of a good map, and we will defer to [Chen and Ruan 2002; 2004] for the analytic setup. With that said, we still must extend the definition above so that it makes sense in a stratified space. We should also note that the orbifold cohomology of Chen and Ruan is not the same as the orbifold cohomology mentioned earlier. This cohomology is simply a way to organize how various classes interact with the stratification of the orbifold. As in the manifold case we start with a compact symplectic orbifold, $Z$, and pick a compatible almost-complex structure, $J$. We then consider moduli spaces of (genus-0) $J$-holomorphic orbicurves into $M$ representing a homology class $A \in H_{2}(Z, \mathbb{Q})$. But we now need to consider a new piece of data which organizes the intersection data so that it is compatible with the stratification. The extra data will be defined by a $k$-tuple $\boldsymbol{x}$, of orbifold strata, $\left(z_{1}, \ldots, z_{k}\right)$. The length $k$ of $\boldsymbol{x}$ should coincide with the number of marked points. We will write such a moduli space as

$$
\mathcal{M}_{0, k}^{A}(\mathcal{Z}, J, x),
$$

and require that the evaluation takes the $j$-th marked point into $z_{j}$. The compactification is similar to the manifold case, and consists of stable maps with the obvious adjustments, the caveat being that we must choose our lift to an orbicurve. After an appropriate construction of cycles as in the manifold case, Chen and Ruan use a virtual cycle construction, so we can define this invariant as in the smooth case above, but we integrate over (the compactification of) $\mathcal{M}_{0, k}^{A}(\mathcal{Z}, J, \boldsymbol{x})$. We will write these invariants

$$
\mathrm{GW}_{A, k, x}^{Z}\left(\alpha_{1}, \ldots, \alpha_{k}\right) \text {. }
$$

Another key difference is that this moduli space differs from the predicted dimension in the smooth case by a factor of $-2 \iota(\boldsymbol{x})$, the so-called degree shifting number. (Again, for the definition see [Chen and Ruan 2002].) The Gromov-Witten invariants satisfy a list of axioms developed by Kontsevich and Manin [1994; 1997]. We will not list all of the axioms, but will mention only some which are used in the text. We use the orbifold notation; for a manifold we would just delete $\boldsymbol{x}$ from the 
notation, setting $\iota(\boldsymbol{x})=0$.

(i) Effective: $\mathrm{GW}_{A, k, x}^{Z}\left(\alpha_{1}, \ldots, \alpha_{n}\right)=0$ as long as $\omega(A)<0$.

(ii) Grading: $\mathrm{GW}_{A, k, x}^{\mathcal{Z}}\left(\alpha_{1}, \ldots, \alpha_{n}\right) \neq 0$ only if

$$
\sum_{j} \operatorname{deg}\left(\alpha_{j}\right)=\operatorname{dim}(z)+2 c_{1}(A)+2 k-6-2 \iota(\boldsymbol{x}) .
$$

(iii) Divisor: Let $\boldsymbol{x}^{j}=\boldsymbol{x}$ with the $j$-th component removed. Suppose that for each component $x_{i}$ of $\boldsymbol{x}$, if $x_{i}$ is mapped into the orbifold singular locus, that stratum is nonsingular as a variety. If $\operatorname{deg}\left(\alpha_{n}\right)=2$ then

$$
\mathrm{GW}_{A, k, x}^{Z}\left(\alpha_{1}, \ldots, \alpha_{n}\right)=\left(\int_{A} \alpha_{n}\right) \mathrm{GW}_{A, k-1, x^{n}}^{Z}\left(\alpha_{1}, \ldots, \alpha_{n-1}\right) .
$$

Now we are in a position to define the Gromov-Witten potential. This is a generating function which gives a formal power series whose coefficients give Gromov-Witten invariants. It is a way to organize all the information from these invariants into one big package. We give the definition here for the manifold case. Pick a basis of $H^{2}(M), a_{1}, \ldots, a_{n}$, for a vector $t$ and a cohomology class $a$, and write $a:=a_{t}=\sum_{i} t_{i} a_{i}$.

Definition A.8. Let $(M, \omega)$ and $J$ be as above. Define the genus-0 Gromov-Witten Potential as

$$
f\left(a_{t}\right)=\sum_{A} \sum_{k} \frac{1}{k !} \mathrm{GW}_{A, k}^{M}\left(a_{t}, \ldots, a_{t}\right) z^{c_{1}(A)} .
$$

The corresponding formula for orbifolds is obtained by accounting for the vector $\boldsymbol{x}$.

\section{Acknowledgements}

Boyer thanks Miguel Abreu, Vestislav Apostolov, David Calderbank, Paul Gauduchon, and Christina Tønnesen-Friedman for helpful discussions on toric geometry. Pati would like to thank Tobias Ekholm, Georgios Dimitroglou Rizell, and Clement Hyrvier for many useful discussions.

\section{References}

[Abreu 2010] M. Abreu, "Kähler-Sasaki geometry of toric symplectic cones in action-angle coordinates", Port. Math. 67:2 (2010), 121-153. MR 2011f:53074 Zbl 1193.53109

[Abreu and Macarini 2012] M. Abreu and L. Macarini, "Contact homology of good toric contact manifolds”, Compos. Math. 148:1 (2012), 304-334. MR 2881318 Zbl 1238.53064

[Audin 1994] M. Audin, "Symplectic and almost complex manifolds", pp. 41-74 in Holomorphic curves in symplectic geometry, edited by M. Audin and J. Lafontaine, Progr. Math. 117, Birkhäuser, Basel, 1994. MR 1274926 
[Banyaga and Molino 1993] A. Banyaga and P. Molino, "Géométrie des formes de contact complètement intégrables de type toriques", pp. 1-25 in Séminaire Gaston Darboux de Géométrie et Topologie Différentielle (Montpellier, 1991-1992), edited by J. Lafontaine, Université Montpellier II, Montpellier, 1993. MR 94e:53029 Zbl 0783.58021

[Barden 1965] D. Barden, "Simply connected five-manifolds", Ann. of Math. (2) 82 (1965), 365-385. MR 32 \#1714 Zbl 0136.20602

[Bourgeois 2002] F. Bourgeois, A Morse-Bott approach to contact homology, PhD thesis, Stanford University, Stanford, CA, 2002, Available at http://search.proquest.com/docview/305591502. MR 2703292

[Bourgeois 2003] F. Bourgeois, "A Morse-Bott approach to contact homology", pp. 55-77 in Symplectic and contact topology: interactions and perspectives (Toronto, ON/Montréal, QC, 2001), edited by Y. Eliashberg et al., Fields Inst. Commun. 35, Amer. Math. Soc., Providence, RI, 2003. MR 2004a:53109 Zbl 1046.57017

[Bourgeois and Mohnke 2004] F. Bourgeois and K. Mohnke, "Coherent orientations in symplectic field theory”, Math. Z. 248:1 (2004), 123-146. MR 2005g:53173 Zbl 1060.53080

[Boyer 2009] C. P. Boyer, "The Sasakian geometry of the Heisenberg group", Bull. Math. Soc. Sci. Math. Roumanie (N.S.) 52(100):3 (2009), 251-262. MR 2011b:53098 Zbl 1199.53102

[Boyer 2011a] C. P. Boyer, "Completely integrable contact Hamiltonian systems and toric contact structures on $S^{2} \times S^{3}$ ", Symmetry Integrability Geom. Methods Appl. 7 (2011), Paper ID \#058. MR 2861218 Zbl 1242.53112 arXiv 1101.5587

[Boyer 2011b] C. P. Boyer, "Extremal Sasakian metrics on $S^{3}$-bundles over $S^{2}$ ", Math. Res. Lett. 18:1 (2011), 181-189. MR 2012d:53132 Zbl 1246.53065

[Boyer 2013] C. P. Boyer, "Maximal tori in contactomorphism groups", Differential Geom. Appl. 31:2 (2013), 190-216. MR 3032643

[Boyer and Galicki 2000a] C. P. Boyer and K. Galicki, "A note on toric contact geometry", J. Geom. Phys. 35:4 (2000), 288-298. MR 2001h:53124 Zbl 0984.53032

[Boyer and Galicki 2000b] C. P. Boyer and K. Galicki, “On Sasakian-Einstein geometry”, Internat. J. Math. 11:7 (2000), 873-909. MR 2001k:53081 Zbl 1022.53038

[Boyer and Galicki 2008] C. P. Boyer and K. Galicki, Sasakian geometry, Oxford University Press, 2008. MR 2009c:53058 Zbl 1155.53002

[Boyer and Tønnesen-Friedman 2013] C. P. Boyer and C. W. Tønnesen-Friedman, "Extremal Sasakian geometry on $T^{2} \times S^{3}$ and related manifolds", Compos. Math. 149:8 (2013), 1431-1456.

[Boyer and Tønnesen-Friedman 2014] C. P. Boyer and C. W. Tønnesen-Friedman, "Extremal Sasakian geometry on $S^{3}$-bundles over Riemann surfaces", Int. Math. Res. Not. 2014:8 (2014), rnt139.

[Boyer et al. 2005] C. P. Boyer, K. Galicki, and J. Kollár, "Einstein metrics on spheres", Ann. of Math. (2) 162:1 (2005), 557-580. MR 2006j:53058 Zbl 1093.53044

[Boyer et al. 2007] C. P. Boyer, K. Galicki, and L. Ornea, "Constructions in Sasakian geometry", Math. Z. 257:4 (2007), 907-924. MR 2008m:53103 Zbl 1134.53021

[Boyer et al. 2008] C. P. Boyer, K. Galicki, and S. R. Simanca, "Canonical Sasakian metrics", Comm. Math. Phys. 279:3 (2008), 705-733. MR 2009a:53077 Zbl 1142.53036

[Boyer et al. 2009] C. P. Boyer, K. Galicki, and S. R. Simanca, "The Sasaki cone and extremal Sasakian metrics", pp. 263-290 in Riemannian topology and geometric structures on manifolds (Albuquerque, NM, 2006), edited by K. Galicki and S. R. Simanca, Progr. Math. 271, Birkhäuser, Boston, 2009. MR 2010e:53078 Zbl 1171.53033 
[Bryant 2001] R. L. Bryant, "Bochner-Kähler metrics", J. Amer. Math. Soc. 14:3 (2001), 623-715. MR 2002i:53096 Zbl 1006.53019

[Calabi 1985] E. Calabi, "Extremal Kähler metrics, II", pp. 95-114 in Differential geometry and complex analysis, edited by I. Chavel and H. M. Farkas, Springer, Berlin, 1985. MR 86h:53067 Zbl 0574.58006

[Chen and Ruan 2002] W. Chen and Y. Ruan, "Orbifold Gromov-Witten theory", pp. 25-85 in Orbifolds in mathematics and physics (Madison, WI, 2001), edited by A. Adem et al., Contemp. Math. 310, Amer. Math. Soc., Providence, RI, 2002. MR 2004k:53145 Zbl 1091.53058

[Chen and Ruan 2004] W. Chen and Y. Ruan, "A new cohomology theory of orbifold", Comm. Math. Phys. 248:1 (2004), 1-31. MR 2005j:57036 Zbl 1063.53091

[Cho et al. 2008] K. Cho, A. Futaki, and H. Ono, "Uniqueness and examples of compact toric SasakiEinstein metrics”, Comm. Math. Phys. 277:2 (2008), 439-458. MR 2008j:53076 Zbl 1144.53058

[Cieliebak et al. 1996] K. Cieliebak, A. Floer, H. Hofer, and K. Wysocki, "Applications of symplectic homology, II: Stability of the action spectrum”, Math. Z. 223:1 (1996), 27-45. MR 97j:58045 Zbl 0869.58013

[Cvetič et al. 2005] M. Cvetič, H. Lü, D. N. Page, and C. N. Pope, "New Einstein-Sasaki spaces in five and higher dimensions”, Phys. Rev. Lett. 95:7 (2005), Article ID \#071101. MR 2006f:53061

[David and Gauduchon 2006] L. David and P. Gauduchon, "The Bochner-flat geometry of weighted projective spaces", pp. 109-156 in Perspectives in Riemannian geometry (Montréal, QC, 2004), edited by V. Apostolov et al., CRM Proc. Lecture Notes 40, Amer. Math. Soc., Providence, RI, 2006. MR 2007h:32032 Zbl 1109.32019

[Eliashberg et al. 2000] Y. Eliashberg, A. Givental, and H. Hofer, "Introduction to symplectic field theory", Geom. Funct. Anal. Special Volume, Part II (2000), 560-673. MR 2002e:53136 Zbl 0989.81114

[Gauduchon 2009] P. Gauduchon, "Hirzebruch surfaces and weighted projective planes", pp. 25-48 in Riemannian topology and geometric structures on manifolds (Albuquerque, NM, 2006), edited by K. Galicki and S. R. Simanca, Progr. Math. 271, Birkhäuser, Boston, 2009. MR 2010h:32023 Zbl 1191.32009

[Gauntlett et al. 2004a] J. P. Gauntlett, D. Martelli, J. Sparks, and D. Waldram, "Sasaki-Einstein metrics on $S^{2} \times S^{3}$ ", Adv. Theor. Math. Phys. 8:4 (2004), 711-734. MR 2006m:53067 Zbl 1136.53317

[Gauntlett et al. 2004b] J. P. Gauntlett, D. Martelli, J. Sparks, and D. Waldram, "Supersymmetric AdS 5 solutions of M-theory”, Classical Quantum Gravity 21:18 (2004), 4335-4366. MR 2006b:83186 Zbl 1059.83038

[Gauntlett et al. 2005] J. P. Gauntlett, D. Martelli, J. Sparks, and D. Waldram, "Supersymmetric AdS backgrounds in string and M-theory", pp. 217-252 in AdS/CFT correspondence: Einstein metrics and their conformal boundaries (Strasbourg, 2003), edited by O. Biquard, IRMA Lect. Math. Theor. Phys. 8, Eur. Math. Soc., Zürich, 2005. MR 2007b:83171 Zbl 1071.81556

[Ghigi and Kollár 2007] A. Ghigi and J. Kollár, "Kähler-Einstein metrics on orbifolds and Einstein metrics on spheres", Comment. Math. Helv. 82:4 (2007), 877-902. MR 2008j:32027 Zbl 1135.53031

[Griffiths and Harris 1978] P. Griffiths and J. Harris, Principles of algebraic geometry, Wiley, New York, 1978. MR 80b:14001 Zbl 0408.14001

[Gromov 1985] M. Gromov, "Pseudoholomorphic curves in symplectic manifolds", Invent. Math. 82:2 (1985), 307-347. MR 87j:53053 Zbl 0592.53025

[Haefliger 1984] A. Haefliger, "Groupoïdes d'holonomie et classifiants", pp. 70-97 in Structure transverse des feuilletages (Toulouse, 1982), edited by J. Pradines, Astérisque 116, Soc. Math. France, Paris, 1984. MR 86c:57026a Zbl 0562.57012 
[Hirzebruch 1951] F. Hirzebruch, "Über eine Klasse von einfach-zusammenhängenden komplexen Mannigfaltigkeiten”, Math. Ann. 124 (1951), 77-86. MR 13,574e Zbl 0043.30302

[Hofer et al. 1996] H. Hofer, K. Wysocki, and E. Zehnder, "Properties of pseudoholomorphic curves in symplectisations, I: Asymptotics", Ann. Inst. H. Poincaré Anal. Non Linéaire 13:3 (1996), 337-379. MR 97e:58029 Zbl 0861.58018

[Hofer et al. 1997] H. Hofer, V. Lizan, and J.-C. Sikorav, "On genericity for holomorphic curves in four-dimensional almost-complex manifolds”, J. Geom. Anal. 7:1 (1997), 149-159. MR 2000d:32045 Zbl 0911.53014

[Karshon 1999] Y. Karshon, "Periodic Hamiltonian flows on four-dimensional manifolds", Mem. Amer. Math. Soc. 141:672 (1999). MR 2000c:53113 Zbl 0982.70011

[Karshon 2003] Y. Karshon, "Maximal tori in the symplectomorphism groups of Hirzebruch surfaces", Math. Res. Lett. 10:1 (2003), 125-132. MR 2004f:53101 Zbl 1036.53063

[Kirwan 1984] F. C. Kirwan, Cohomology of quotients in symplectic and algebraic geometry, Mathematical Notes 31, Princeton University Press, 1984. MR 86i:58050 Zbl 0553.14020

[Kontsevich and Manin 1994] M. Kontsevich and Y. Manin, "Gromov-Witten classes, quantum cohomology, and enumerative geometry”, Comm. Math. Phys. 164:3 (1994), 525-562. MR 95i:14049 Zbl 0853.14020

[Kontsevich and Manin 1997] M. Kontsevich and Y. Manin, "Gromov-Witten classes, quantum cohomology, and enumerative geometry", pp. 607-653 in Mirror symmetry, II, edited by B. Greene and S.-T. Yau, AMS/IP Stud. Adv. Math. 1, Amer. Math. Soc., Providence, RI, 1997. MR 1416351 Zbl 0931.14030

[Lafontaine 1981] J. Lafontaine, "Surfaces de Hirzebruch", pp. 61-76 in Riemannian geometry in dimension 4 (Paris, 1978-1979), edited by L. Bérard Bergery et al., Textes Math. 3, CEDIC, Paris, 1981. MR 769131 Zbl 0482.14013

[Legendre 2011a] E. Legendre, "Existence and non-uniqueness of constant scalar curvature toric Sasaki metrics”, Compos. Math. 147:5 (2011), 1613-1634. MR 2834736 Zbl 1237.53039

[Legendre 2011b] E. Legendre, "Toric geometry of convex quadrilaterals", J. Symplectic Geom. 9:3 (2011), 343-385. MR 2012g:53079 Zbl 1233.14032

[Lerman 2003a] E. Lerman, “Contact toric manifolds”, J. Symplectic Geom. 1:4 (2003), 785-828. MR 2004m:53147 Zbl 1079.53118

[Lerman 2003b] E. Lerman, "Maximal tori in the contactomorphism groups of circle bundles over Hirzebruch surfaces”, Math. Res. Lett. 10:1 (2003), 133-144. MR 2004g:53097 Zbl 1042.53059

[Lerman 2004] E. Lerman, "Homotopy groups of $K$-contact toric manifolds", Trans. Amer. Math. Soc. 356:10 (2004), 4075-4083. MR 2005b:53136 Zbl 1057.53062

[Lerman and Tolman 1997] E. Lerman and S. Tolman, "Hamiltonian torus actions on symplectic orbifolds and toric varieties", Trans. Amer. Math. Soc. 349:10 (1997), 4201-4230. MR 98a:57043 Zbl 0897.58016

[Martelli and Sparks 2005] D. Martelli and J. Sparks, "Toric Sasaki-Einstein metrics on $S^{2} \times S^{3}$ ", Phys. Lett. B 621:1-2 (2005), 208-212. MR 2006c:53041 Zbl 1247.53055

[McDuff and Salamon 2004] D. McDuff and D. Salamon, J-holomorphic curves and symplectic topology, American Mathematical Society Colloquium Publications 52, Amer. Math. Soc., Providence, RI, 2004. MR 2004m:53154 Zbl 1064.53051

[Ness 1984] L. Ness, "A stratification of the null cone via the moment map", Amer. J. Math. 106:6 (1984), 1281-1329. MR 86c:14010 Zbl 0604.14006 
[Pati 2009] J. Pati, "Contact homology of $S^{1}$-bundles over some symplectically reduced orbifolds", preprint, 2009. arXiv 0910.5934

[Pati 2010] J. Pati, Contact homology of toric contact manifolds of Reeb type, $\mathrm{PhD}$ thesis, University of New Mexico, Albuquerque, NM, 2010, Available at http://search.proquest.com/docview/757731908. MR 2792890

[Robbin and Salamon 1993] J. Robbin and D. Salamon, "The Maslov index for paths", Topology 32:4 (1993), 827-844. MR 94i:58071 Zbl 0798.58018

[Sparks 2011] J. Sparks, "Sasaki-Einstein manifolds", pp. 265-324 in Geometry of special holonomy and related topics, edited by N. C. Leung and S.-T. Yau, Surv. Differ. Geom. 16, International Press, Somerville, MA, 2011. MR 2012k:53082 Zbl 1256.53037

[Tanno 1970] S. Tanno, "On the isometry groups of Sasakian manifolds", J. Math. Soc. Japan 22 (1970), 579-590. MR 42 \#6755 Zbl 0197.48004

Received April 23, 2012. Revised July 4, 2013.

Charles P. BOYER

Department OF MATHEMATICS AND StATISTICS

UNIVERSITY OF NEW MEXICO

222 SMLC

ALBUQUERQUE, NM 87131

UNITED STATES

cboyer@math.unm.edu

JUSTIN PATI

MATEMATISKA INSTITUTIONEN

UPPSALA UNIVERSITET

BOX 480

SE-751 06 UPPSALA

SWEDEN

justin@math.uu.se 


\title{
PACIFIC JOURNAL OF MATHEMATICS
}

\author{
msp.org/pjm
}

Founded in 1951 by E. F. Beckenbach (1906-1982) and F. Wolf (1904-1989)

\section{EDITORS}

V. S. Varadarajan (Managing Editor)

Department of Mathematics

University of California

Los Angeles, CA 90095-1555

pacific@math.ucla.edu

Paul Balmer

Department of Mathematics

University of California

Los Angeles, CA 90095-1555

balmer@math.ucla.edu

Daryl Cooper

Department of Mathematics

University of California

Santa Barbara, CA 93106-3080 cooper@math.ucsb.edu

Jiang-Hua $\mathrm{Lu}$

Department of Mathematics

Pokfulam Rd., Hong Kong jhlu@maths.hku.hk
The University of Hong Kong

Don Blasius

Department of Mathematics University of California

Los Angeles, CA 90095-1555

blasius@math.ucla.edu

Robert Finn

Department of Mathematics Stanford University

Stanford, CA 94305-2125

finn@math.stanford.edu

Sorin Popa

Department of Mathematics

University of California

Los Angeles, CA 90095-1555 popa@math.ucla.edu

Paul Yang

Department of Mathematics Princeton University

Princeton NJ 08544-1000

yang@math.princeton.edu

\section{PRODUCTION}

Silvio Levy, Scientific Editor, production@msp.org

\section{SUPPORTING INSTITUTIONS}

ACADEMIA SINICA, TAIPEI

CALIFORNIA INST. OF TECHNOLOGY

INST. DE MATEMÁTICA PURA E APLICADA

KEIO UNIVERSITY

MATH. SCIENCES RESEARCH INSTITUTE

NEW MEXICO STATE UNIV.

OREGON STATE UNIV.

\author{
STANFORD UNIVERSITY \\ UNIV. OF BRITISH COLUMBIA \\ UNIV. OF CALIFORNIA, BERKELEY \\ UNIV. OF CALIFORNIA, DAVIS \\ UNIV. OF CALIFORNIA, LOS ANGELES \\ UNIV. OF CALIFORNIA, RIVERSIDE \\ UNIV. OF CALIFORNIA, SAN DIEGO \\ UNIV. OF CALIF., SANTA BARBARA
}

\author{
Vyjayanthi Chari \\ Department of Mathematics \\ University of California \\ Riverside, CA 92521-0135 \\ chari@math.ucr.edu \\ Kefeng Liu \\ Department of Mathematics \\ University of California \\ Los Angeles, CA 90095-1555 \\ liu@math.ucla.edu \\ Jie Qing \\ Department of Mathematics \\ University of California \\ Santa Cruz, CA 95064 \\ qing@cats.ucsc.edu
}

These supporting institutions contribute to the cost of publication of this Journal, but they are not owners or publishers and have no responsibility for its contents or policies.

See inside back cover or msp.org/pjm for submission instructions.

The subscription price for 2014 is US \$410/year for the electronic version, and \$535/year for print and electronic.

Subscriptions, requests for back issues and changes of subscribers address should be sent to Pacific Journal of Mathematics, P.O. Box 4163, Berkeley, CA 94704-0163, U.S.A. The Pacific Journal of Mathematics is indexed by Mathematical Reviews, Zentralblatt MATH, PASCAL CNRS Index, Referativnyi Zhurnal, Current Mathematical Publications and Web of Knowledge (Science Citation Index).

The Pacific Journal of Mathematics (ISSN 0030-8730) at the University of California, c/o Department of Mathematics, 798 Evans Hall \#3840, Berkeley, CA 94720-3840, is published twelve times a year. Periodical rate postage paid at Berkeley, CA 94704, and additional mailing offices. POSTMASTER: send address changes to Pacific Journal of Mathematics, P.O. Box 4163, Berkeley, CA 94704-0163.

PJM peer review and production are managed by EditFLOW ${ }^{\circledR}$ from Mathematical Sciences Publishers.

\section{PUBLISHED BY}

mathematical sciences publishers

nonprofit scientific publishing

http://msp.org/

(C) 2014 Mathematical Sciences Publishers 


\section{PACIFIC JOURNAL OF MATHEMATICS}

Volume $267 \quad$ No. $2 \quad$ February 2014

Sums of squares in algebraic function fields over a complete discretely valued field

KARIM JOHANNES BECHER, DAVID GRIMM and JAN VAN GEEL

On the equivalence problem for toric contact structures on $\mathbf{S}^{\mathbf{3}}$-bundles over $S^{2}$

\section{Charles P. Boyer and Justin PATi}

An almost-Schur type lemma for symmetric $(2,0)$ tensors and applications

Xu CHENG

Algebraic invariants, mutation, and commensurability of link complements

ERIC CHESEBRO and JASON DEBLOIS

Taut foliations and the action of the fundamental group on leaf spaces and universal circles

YOSUKE KANO

A new monotone quantity along the inverse mean curvature flow in $\mathbb{R}^{n}$

KwoK-Kun Kwong and Pengzi Miao

Nonfibered L-space knots

TYE LIDMAN and LIAM WATSON

Families and Springer's correspondence

GEORGE LUSZTIG

Reflexive operator algebras on Banach spaces

Florence Merlevède, Costel Peligrad and Magda Peligrad Harer stability and orbifold cohomology

NiCOLA PAGANI

Spectra of product graphs and permanents of matrices over finite rings

LE ANH VINH

The concavity of the Gaussian curvature of the convex level sets of minimal surfaces with respect to the height

PEI-HE WANG 